\title{
LA PÉSIMA ECONOMÍA NEOCLÁSICA DEL CAMBIO CLIMÁTICO*
}

Steve Keen ${ }^{\mathrm{a}}$

" DOI: https://doi.org/10.18601/01245996.v23n44.02. Recepción: 15-052020, aceptación: 27-11-2020. Sugerencia de citación: Keen, S. (2021). La pésima economía neoclásica del cambio climático. Revista de Economía Institucional, 23(44), 13-52. Artículo original: Steve Keen (2020) The appallingly bad neoclassical economics of climate change, Globalizations, [DOI: 10.1080/14747731.2020.1807856]. Traducción de Alberto Supelano; se publica con las autorizaciones correspondientes.

a Investigador distinguido, Instituto de Estrategia, Resiliencia y Seguridad (ISRS), University College London, Londres, UK, [s.keen@isrs.org.uk], [http://orcid.org/0000-0002-0439-1809]. 


\section{La pésima economía neoclásica del cambio climático}

Resumen Las predicciones de los economistas del daño a la economía causado por el cambio climático son demasiado optimistas en comparación con las advertencias de los científicos sobre el daño a la biosfera. Esto se debe ante todo a que los economistas predicen los daños usando tres métodos espurios: suponen que un $90 \%$ del PIB no será afectado por el cambio climático porque ocurre puertas adentro; usan la relación actual entre temperatura y PIB como proxy del impacto del calentamiento global, y encuestas que diluyen las advertencias extremas de los científicos con las expectativas optimistas de los economistas. Nordhaus ha malinterpretado la literatura científica para justificar el uso de una función suave que describa el daño del cambio climático al PIB. Cuando se corrigen estos errores los daños pueden ser al menos un orden de magnitud peores que los que predicen los economistas y tan graves que amenazan la supervivencia de la civilización humana.

Palabras clave: PIB, calentamiento global, cambio climático; JEL: D31, E44; Q51

\section{The appallingly bad neoclassical economics of climate change}

Abstract Forecasts by economists of the economic damage from climate change have been notably sanguine, compared to warnings by scientists about damage to the biosphere. This is because economists made their own predictions of damages, using three spurious methods: assuming that about $90 \%$ of GDP will be unaffected by climate change, because it happens indoors; using the relationship between temperature and GDP today as a proxy for the impact of global warming over time; and using surveys that diluted extreme warnings from scientists with optimistic expectations from economists. Nordhaus has misrepresented the scientific literature to justify the using a smooth function to describe the damage to GDP from climate change. Correcting for these errors makes it feasible that the economic damages from climate change are at least an order of magnitude worse than forecast by economists, and may be so great as to threaten the survival of human civilization.

Keywords: PIB, global warming, change; JEL: D31, E44; Q51

\section{A péssima economia neoclássica da mudança climática}

Resumo As previsões dos economistas sobre danos à economia devido à mudança climática são excessivamente otimistas em comparação com as advertências dos cientistas sobre os danos à biosfera. Isso ocorre principalmente porque os economistas prevêem os danos usando três métodos espúrios: eles presumem que $90 \%$ do PIB não será afetado pelas mudanças climáticas porque elas ocorrem em ambientes fechados; eles usam a relação atual entre temperatura e PIB como um proxy para o impacto do aquecimento global e pesquisas que diluem os alertas extremos dos cientistas com as expectativas otimistas dos economistas. Nordhaus interpretou mal a literatura científica para justificar o uso de uma função suave para descrever os danos da mudança climática ao PIB. Quando esses erros são corrigidos, o dano pode ser pelo menos uma ordem de magnitude pior do que os economistas prevêem e tão grave que ameaça a sobrevivência da civilização humana.

Palavras-chave: PIB, aquecimento global, mudança; JEL: D31, E44; Q51 
$\mathrm{W}$ illiam Nordhaus recibió en 2018 el Premio del Banco de Suecia en Ciencias Económicas en memoria de Alfred Nobel (Mirowski, 2020) por su obra sobre el cambio climático. Su primer trabajo importante en esta área fue "World dynamics: Measurement without data" (Nordhaus, 1973), que atacó las predicciones pesimistas de Jay Forrester en World dynamics (Forrester, 1971, 1973), entre otras cosas, porque sus predicciones no se basaban firmemente en la investigación empírica:

E1 tratamiento de las relaciones empíricas en World Dynamics se puede resumir como medición sin datos [...] No toma ni una sola relación o variable de datos reales o estudios empiricos (Nordhaus, 1973, p. 1157, cursivas del original. Se añadieron las cursivas posteriores).

No hay ninguna referencia explicita o clara a datos o estudios empíricos existentes (ibíd., p. 1182).

Mientras que la mayoría de los científicos requerirían la validación empírica de los supuestos o las predicciones del modelo antes de declarar su contenido de verdad, Forrester parece contentarse con la plausibilidad subjetiva [...] (ibíd., p. 1183).

Sexto, hay falta de humildad para predecir el futuro. ¿Podemos considerar seriamente las predicciones de Forrester (o de cualquiera) en economía y ciencias sociales para los próximos 130 años? En general, las predicciones económicas de largo plazo han sido muy deficientes [...] Y ahora, sin la más escasa referencia a la teoría económica o a datos empíricos, Forrester predice que el nivel de vida material del mundo llegará al pico en 1990 y luego disminuirá (ibíd., p. 1183).

Después de este artículo, la investigación de Nordhaus se centró en la economía del cambio climático. Por su crítica a Forrester, cabría esperar con razón que Nordhaus fuera escrupuloso y basara su modelación en datos empíricos sólidos.

Esa expectativa se vería frustrada. Mientras que Nordhaus calificó el trabajo de Forrester como "medición sin datos", el de Nordhaus se puede calificar como "invención de números para respaldar una creencia preexistente": en concreto, que el cambio climático solo puede tener un impacto trivial en la economía. Esta práctica fue imitada, en vez de ser cuestionada, por economistas neoclásicos posteriores; con honrosas excepciones como Pindyck (2017), Weitzman (2011a y b), DeCanio (2003), Cline (1996), Darwin (1999), Kaufmann (1997 y 1998) y Quiggin y Horowitz (1999).

El producto final es un conjunto de supuestas estimaciones empíricas del impacto del cambio climático en la economía que son espúreas $\mathrm{y}$, sin embargo, se usan para calibrar los modelos de evaluación integrada (MEI) que suelen guiar las respuestas de política 
al cambio climático. DeCanio expresó muy bien el significado y el peligro de esta labor en su libro Modelos económicos del cambio climático: una crítica:

Quizás la mayor amenaza del cambio climático es el riesgo que impone por la catastrófica disrupción a gran escala de los sistemas terrestres [...]

La práctica usual equivale a realizar un experimento único e irreversible de resultado desconocido con la habitabilidad de todo el planeta.

Dada la magnitud de lo que está en juego, es sorprendente que gran parte del debate sobre el clima se haya realizado en términos de economía [...]

No obstante, es innegable que los argumentos, afirmaciones y cálculos económicos han sido la influencia dominante en el debate político sobre la política climática en Estados Unidos y en todo el mundo [...] Es una pregunta abierta si los argumentos económicos fueron la causa o solo una justificación ex post de las decisiones tomadas por ambas administraciones, pero no hay duda de que los economistas pretenden que sus cálculos dicten el curso de acción adecuado (DeCanio, 2003, pp. 2-4).

El impacto de estos economistas va más allá de la simple asesoría a los gobiernos, pues redactan los componentes económicos de los informes formales del Panel Intergubernamental sobre Cambio Climático (IPCC), la principal autoridad que coordina la respuesta de la humanidad al cambio climático. Las displicentes conclusiones a las que llegan -como las siguientes, tomadas del Informe IPCC 2014 (Field et al., 2014), tienen mucho más peso entre los políticos, obsesionados por las tasas de crecimiento del PIB de sus países, que las alarmantes advertencias ecológicas de las secciones del Informe redactadas por científicos reales:

Es difícil estimar los impactos económicos globales del cambio climático. Las estimaciones del impacto económico completadas en los últimos 20 años varían en su cobertura de subconjuntos de sectores económicos y dependen de un gran número de supuestos, muchos de ellos discutibles; y muchas estimaciones no tienen en cuenta cambios catastróficos, puntos de inflexión y muchos otros factores. Con estas limitaciones reconocidas, las estimaciones incompletas de las pérdidas económicas anuales globales por un aumento de temperatura adicional de $\sim 2{ }^{\circ} \mathrm{C}$ están entre un 0,2 y un 2,0\% del ingreso (Arent et al., 2014, p. 663, cursivas añadidas).

Esto no predice una caída de la tasa anual de crecimiento económico, que sería significativa incluso en el límite inferior del 0,2\%; predice que el nivel del PIB será de un $0,2 \%$ a un $2 \%$ menor, cuando la temperatura global sea $2{ }^{\circ} \mathrm{C}$ mayor que los niveles preindustriales, en comparación con lo que sería en ausencia total de calentamiento global. Esto implica una disminución trivial de la tasa de crecimiento 
económico prevista entre 2014 y cuando se produzca el aumento de $2{ }^{\circ} \mathrm{C}$, incluso en el límite superior del $2 \%$.

Dado el impacto de los economistas en la política pública hacia el cambio climático, y la inmediatez de la amenaza del cambio climático que hoy enfrentamos (Amen et al., 2008; Gills y Morgan, 2019; Gills, 2019), ese trabajo pronto podría ser expuesto como el engaño más significativo y peligroso en la historia de la ciencia.

\section{EMPÍRICOS FICTICIOS}

Las relaciones numéricas que según los economistas existen entre el cambio de la temperatura global y el cambio del PIB se resumieron en la tabla 1 del capítulo "Sectores y servicios económicos clave" (Arent et al., 2014b) del Informe IPCC 2014, Cambio climático 2014: impactos, adaptación y vulnerabilidad (Field et al., 2014), las cuales se reproducen en la gráfica 1.

Gráfica 1

Estimaciones del impacto total del cambio climático

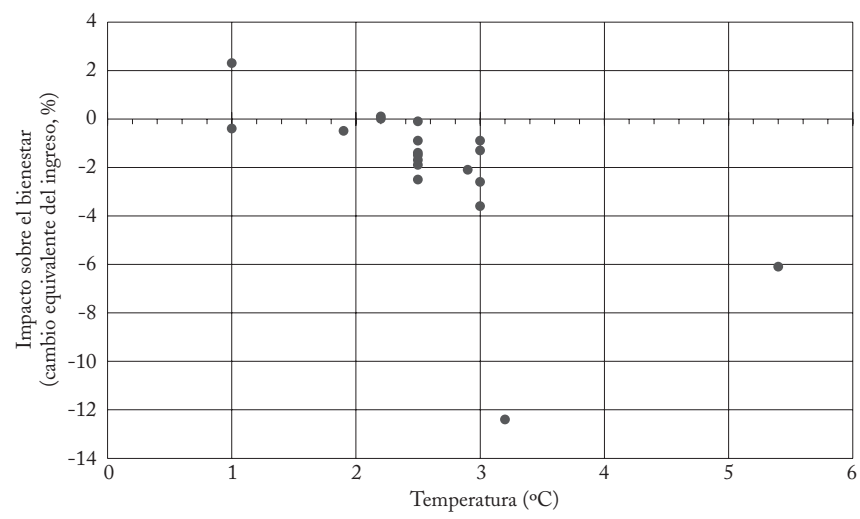

Fuente: Arent et al. (2014b).

Las fuentes de estos números - como explico más adelante, no se pueden llamar "puntos de datos"- se mencionan en la Tabla SM10-1 del suplemento de ese informe (Arent et al., 2014, p. SM 10-4) ${ }^{1}$. El IPCC clasifica los enfoques utilizados en cuatro grupos: "Enumeración" (10 estudios); "Estadístico" (5 estudios); "EGC” (equilibrio

${ }^{1}$ Se reproduce en el cuadro 3 del anexo, al que se añaden Nordhaus (1991), los estudios empíricos adicionales ubicados por Nordhaus y Moffat (2017) y una metodología adicional. 
general computable: 2 estudios; uno con 2 resultados); y "Solicitud a expertos" (1 estudio).

\section{ENUMERACIÓN: LO QUE NO CUENTA ES LO QUE CUENTA}

La anodina descripción de Tol de lo que implica el enfoque de "Enumeración" hace que parezca inobjetable:

En este enfoque, las estimaciones de los "efectos físicos" del cambio climático se obtienen, una por una, de artículos de ciencias naturales, los que a su vez se pueden basar en una combinación de modelos climáticos, modelos de impacto y experimentos de laboratorio. A los impactos físicos se les debe dar un precio, y luego se suman. Para los productos agrícolas, un ejemplo de un bien o servicio comercializado, se utilizan artículos de agronomía para predecir el efecto del clima en el rendimiento de los cultivos, y después se usan precios de mercado o modelos económicos para valorar el cambio del producto (Tol, 2009, pp. 31-32).

Pero este análisis se inició desde la perspectiva, enunciada en la primera referencia de esta tradición (Nordhaus, 1991), de que el cambio climático es un tema relativamente trivial:

Primero se debe reconocer que las sociedades humanas prosperan en una amplia variedad de zonas climáticas. En la mayor parte de la actividad económica, variables no climáticas como la capacidad laboral, el acceso a los mercados o la tecnología opacan las consideraciones climáticas en la determinación de la eficiencia económica (ibíd., p. 930; cursivas añadidas).

Si en ese tiempo hubiese existido un proceso decente de evaluación de la investigación del impacto económico del cambio climático, ese párrafo debería haber hecho sonar las alarmas: sí, es muy probable que el clima sea hoy un determinante menos importante de la "eficiencia económica actual que "la capacidad laboral, el acceso a los mercados o la tecnología" cuando se compara una región o un país con otro boy en día. Pero, ¿̇cuál es la relevancia de esta comparación de corte transversal para evaluar el impacto de la drástica alteración del clima en todo el planeta a través del tiempo, mediante la retención de energía solar adicional por los gases de efecto invernadero adicionales?

Una frase más adelante, Nordhaus excluye de toda consideración al $87 \%$ de la industria estadounidense, con base en que tiene lugar "en ambientes cuidadosamente controlados que no serán afectados directamente por el cambio climático":

La tabla 5 muestra un desglose sectorial del ingreso nacional de Estados Unidos, en el que la economía se subdivide por la sensibilidad sectorial al calentamiento por efecto invernadero. Es probable que los sectores más sensibles sean aquellos, como la agricultura y la silvicultura, en los 
que el producto depende en forma significativa de variables climáticas. En el otro extremo están actividades, como la cirugía cardiovascular o la fabricación de microprocesadores en "salas esterilizadas", que se realizan en ambientes cuidadosamente controlados que no serán afectados directamente por el cambio climático. Nuestra estimación es que un 3\% del producto nacional de Estados Unidos proviene de sectores muy sensibles, otro $10 \%$ de sectores moderadamente sensibles y un $87 \%$ de sectores infimamente afectados por el cambio climático (ibíd., p. 930, cursivas añadidas).

Parece razonable que los ejemplos de "cirugía cardiovascular o fabricación de microprocesadores en 'salas esterilizadas" son actividades que se llevan a cabo en "entornos cuidadosamente controlados". Pero la lista de Nordhaus de las industrias que él simplemente supuso que serían ínfimamente afectadas por el cambio climático es tan amplia, y tan extensa, que es obvio que lo que quiso decir con "no ser afectadas directamente por el cambio climático" es todo lo que tiene lugar puertas adentro, o incluso en subterráneos, pues incluye la minería como uno de los sectores no afectados. El cuadro 1 es un extracto de su desglose de la actividad económica por la vulnerabilidad al cambio climático en dólares de 1991.

Cuadro 1

\begin{tabular}{lcc}
\hline Sector & $\begin{array}{c}\text { Valor } \\
\text { (miles de millones) }\end{array}$ & $\begin{array}{c}\text { Porcentaje } \\
\text { del total }\end{array}$ \\
\hline Efecto insignificante & & \\
Manufactura y minería & 627,4 & 26,0 \\
Otros transportes y comunicaciones & 132,6 & 5,5 \\
Finanzas, seguros y saldo inmobiliario & 274,8 & 11,4 \\
Servicios comerciales y otros & 674,6 & 27,9 \\
Servicios gubernamentales & 337,0 & 14,0 \\
Resto del mundo & 50,3 & 2,1 \\
Total "efecto insignificante” & $2.096,7$ & 86,9 \\
\hline
\end{tabular}

Fuente: Nordhaus (1991, Tabla 5, p. 931).

Puesto que este fue el primer artículo de una tradición de investigación, cabría esperar que los investigadores posteriores cuestionaran este supuesto. Sin embargo, en vez de cuestionarlo, lo replicaron. El Informe IPCC 2014 repite que el cambio climático será un determinante trivial del desempeño económico futuro:

En la mayoría de los sectores económicos, el impacto del cambio climático será pequeño con respecto al impacto de otros factores (evidencia media, alto nivel de acuerdo). Los cambios de población, edad, ingreso, tecnología, precios relativos, estilo de vida, regulación, gobernanza y muchos otros aspectos del desarrollo socioeconómico tendrán mayor impacto en 
la oferta y la demanda de bienes y servicios económicos que el cambio climático (Arent et al., 2014, p. 662).

También repite que las actividades puertas adentro no serán afectadas. El único cambio entre Nordhaus 1991 y el Informe del IPCC 23 años después es que ya no se clasifica a la minería en el grupo "no realmente expuesto al cambio climático" (Nordhaus, 1993)². En cambio, repite el supuesto de Nordhaus de que todo lo que se haga puertas adentro no será afectado por el cambio climático:

\section{Preguntas frecuentes $10.3 \mid$ ¿Otros sectores económicos son también vulnerables al cambio climático?}

Actividades económicas como la agricultura, la silvicultura, la pesca y la minería están expuestas al estado del tiempo y, por tanto, son vulnerables al cambio climático. Otras actividades económicas, como la manufactura y los servicios, se realizan sobre todo en ambientes controlados y no están realmente expuestas al cambio climático (Arent et al., 2014b, p. 688; cursivas añadidas).

Todos los artículos intermedios entre Nordhaus 1991 e IPCC 2014 mantienen este supuesto: ni la manufactura, la minería, el transporte, las comunicaciones, las finanzas, los seguros y los bienes raíces no costeros, ni el comercio minorista y mayorista, ni los servicios del gobierno aparecen en las industrias "enumeradas" en la columna Cobertura del cuadro 3 del anexo. Todos esos estudios simplemente supusieron que estas industrias, que representan cerca del $90 \%$ del PIB, no serán afectadas por el cambio climático.

En las preguntas frecuentes antes citadas aparece el "cuento del jugador de póquer”, que implica que estos economistas neoclásicos están a la par del presidente de Estados Unidos Donald Trump en su apreciación de lo que implica el cambio climático: la afirmación de que "actividades económicas como la agricultura, la silvicultura, la pesca y la minería están expuestas al estado del tiempo y, por tanto, son vulnerables al cambio climático". Así, dicen explícitamente que si una actividad está expuesta al clima, es vulnerable al cambio climático, y si no lo está, "no está realmente expuesta al cambio climático". Equiparan el clima con el estado del tiempo.

${ }^{2}$ Quizás esto fue una concesión al hecho de que hoy muchas minas son a cielo abierto. En 1993, Nordhaus señaló específicamente que la "minería subterránea" estaba a salvo del cambio climático: "En realidad, la mayor parte de la economía estadounidense tiene poca interacción directa con el clima [...] Más en general, la minería subterránea, la mayoría de los servicios, las comunicaciones y la manufactura no serán muy afectadas por el cambio climático; sectores que abarcan cerca del 85\% del PIB" (Nordhaus 1993, p. 15). Dicho esto, ninguno de los estudios de "enumeración" consideró realmente el impacto del cambio climático en la minería; ver el cuadro 3 del anexo. 
Aunque este es un juicio severo sobre los colegas académicos, no hay otra manera de dar sentido a su decisión colectiva de excluir, por sus supuestos, casi el 90\% del PIB de su enumeración de los daños por el cambio climático. Tampoco hay otra manera de interpretar el supuesto central de su otro método dominante, el de componer números para sus modelos, al que llaman método "estadístico" o "de corte transversal".

\section{EL “ENFOQUE ESTADÍSTICO”}

Aunque encontrar la falla fundamental en el enfoque de "enumeración” requirió investigación adicional, la falla del enfoque estadístico era evidente en la primera referencia que leí sobre él, el artículo "Los efectos económicos del cambio climático” de Richard Tol (2014), muy corregido y muy criticado (Gelman, 2014, 2015, 2019; Nordhaus y Moffat, 2017, p. 10):

Un enfoque alternativo, ejemplificado en el trabajo de Mendelsohn (Mendelsohn, Morrison et al., Mendelsohn, Schlesinger et al., 2000) se puede llamar enfoque estadístico. Este Se basa en estimaciones directas de los impactos en el bienestar que usan variaciones observadas (en el espacio, dentro de un solo pais) de precios y gastos para discernir el efecto del clima. Mendelsohn supone que la variación observada de la actividad económica con respecto al clima en el espacio también se mantiene en el tiempo; y usa modelos climáticos para estimar el efecto futuro del cambio climático (Tol, 2009, p. 32).

Si bien la falacia metodológica en este razonamiento no es clara de inmediato - puesto que numerosos árbitros académicos han dejado pasar artículos que adoptan este supuesto-, vale la pena pensar qué significaría si fuese correcto.

En Estados Unidos es cierto en general que las regiones muy cálidas y muy frías tienen un nivel de ingreso per cápita menor que las de temperatura media. Así, estados continentales contiguos de esas regiones - por ejemplo Florida (temperatura media $22,5^{\circ} \mathrm{C}$ ) y Dakota del Norte (temperatura media $4,7^{\circ} \mathrm{C}$ ) - tienen ingresos per cápita menores que Nueva York (temperatura media $7,4^{\circ} \mathrm{C}$ ). Pero la diferencia de temperaturas medias está lejos de ser la única razón de las diferencias de ingreso, y en el esquema de cosas más amplio las diferencias son de todos modos triviales: en cuanto estados americanos, a nivel global todos están en el rango alto de ingreso per cápita (26.000, 26.700 y 43.300 dólares anuales de 2000, respectivamente). En un estudio estadístico de la relación entre Producto Estatal Bruto (PEB) per cápita y temperatura se encontrará, por tanto, una relación débil y no lineal, en la que el PEB per cápita aumenta desde 
temperaturas bajas, llega al pico a temperaturas medias y disminuye a temperaturas más altas.

Si luego se supone que esta misma relación entre PIB y temperatura se presenta cuando las temperaturas globales aumentan con el calentamiento global, se concluirá que el calentamiento global tiene un impacto trivial en el PIB global. La suposición es la conclusión.

Esto se ilustra en la gráfica 2, que muestra un diagrama de dispersión de las desviaciones de la temperatura promedio nacional por estado en ${ }^{\circ} \mathrm{C}$, contra las desviaciones del promedio nacional (PIB per cápita) del Producto Estatal Bruto per cápita como porcentaje del PIB (los datos se encuentran en el cuadro 4 del anexo), y un ajuste cuadrático de esos datos que tiene un coeficiente de $-0,00318^{3}, \mathrm{y}$, como se esperaba, un coeficiente de correlación débil de $0,31^{4}$.

Gráfica 2

Correlación entre temperatura y Producto Estatal Bruto per cápita Estados Unidos

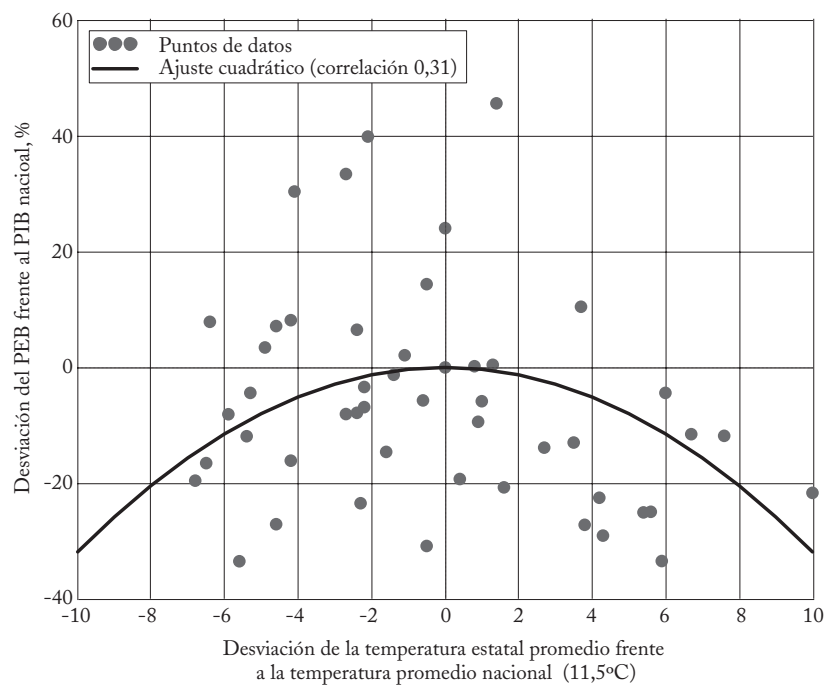

Fuente: cuadro 4.

3 De hecho, este es muy cercano al coeficiente que utilizó Nordhaus en su función de daño en 1999, y mayor que el que usa desde 2008, como se analiza en la página 57.

${ }^{4}$ Estos datos son una amalgama de la temperatura promedio por estado en 1971-2000, el PIB real en 2000 y la población en 2010. Sin embargo, se aplicarían resultados similares a un conjunto de datos más coherente, y el resultado de la regresión derivado de ellos solo tiene fines ilustrativos. 
Esta regresión produce un modelo "en muestra" muy deficiente, pero no del todo inútil, de la ligera influencia de las desviaciones de temperatura promedio de Estados Unidos en las desviaciones de su PIB per cápita promedio. En términos más sencillos, la ecuación (1) dice que el Producto Estatal Bruto per cápita cae el 0,318\% (del PIB per cápita promedio nacional) por cada ${ }^{\circ} \mathrm{C}$ de diferencia de temperatura (de la temperatura promedio nacional) al cuadrado:

$G S P_{P C}(\Delta T)=-0,318 \% \Delta T^{2}$

Una absurda recomendación de política "fuera de muestra" de este modelo sería que el PIB de Estados Unidos aumentaría si los estados más cálidos y más fríos se acercaran a la temperatura promedio de Estados Unidos. Esta absurda recomendación se podría "refinar" usando estos mismos datos para calcular la temperatura óptima para el PIB de Estados Unidos y luego proponer que todos los estados se acerquen a esa temperatura. Esta "política" es, por supuesto, claramente imposible, porque Estados no puede modificar su ubicación en el planeta.

Pero los economistas que hicieron estos estudios razonaron que el calentamiento global lograría el mismo resultado con el tiempo (con el inconveniente de que se aplicaría por igual a todas las regiones). De modo que sí calcularon las temperaturas óptimas para cada uno de los sectores que esperaban ver afectados por el cambio climático; $y$ sus cálculos excluyeron la misma lista de sectores que el enfoque de "enumeración" supuso que no serian afectados (manufactura, mineria, servicios, etc.):

La función de respuesta en forma reducida y la de corte transversal implican que la productividad neta de los sectores económicos sensibles es una función de la temperatura con forma de colina (Mendelsohn, Schlesinger et al., 2000). El calentamiento produce beneficios a los países que hoy se encuentran en el lado frío de la colina y perjudica a los países del lado cálido de la colina. La temperatura óptima exacta varía según el sector. Por ejemplo, según el modelo ricardiano, las temperaturas óptimas para la agricultura, la silvicultura y la energía son de 14,2, 14,8 y $8,6{ }^{\circ} \mathrm{C}$, respectivamente. Con el modelo en forma reducida, las temperaturas óptimas para la agricultura y la energía son de 11,7 y $10,0{ }^{\circ} \mathrm{C}$ (Mendelsohn, Morrison et al. 2000, p. 558).

Luego estimaron el impacto del aumento de la temperatura global en el PIB, suponiendo los mismos coeficientes que encontraron para las relaciones entre temperatura y producto actuales (usando lo que Tol llamó enfoque "estadístico" y Mendelsohn llama con más precisión enfoque "de corte transversal") se podian usar para estimar el impacto del calentamiento global. Esto llevó a más de un estudio que concluía 
que el aumento de las temperatura global por el calentamiento global beneficiaría a la economía. Por ejemplo, al de Mendelsohn, Schlesinger et al. (2000) sobre el impacto de un aumento de $2,5^{\circ} \mathrm{C}$ en la temperatura global:

En comparación con el tamaño de la economía en 2100 (217 billones de dólares), los efectos del mercado son pequeños [...] Las funciones de respuesta climática de corte transversal implican un rango más estrecho de impactos entre GCM: de 97 a 185 mil millones de dólares de beneficios, con un promedio de 145 mil millones de dólares de beneficios al año (Mendelsohn, Schlesinger et al., 2000, p. 41, cursivas añadidas).

Una vez más, el supuesto explícito de estos economistas es que no importa cómo se altere la temperatura. Bien sea que esto se haga hipotéticamente alterando la ubicación de una región en el planeta, lo que es imposible, o alterando la temperatura de todo el planeta -que es lo que está haciendo el cambio climático- supusieron que el impacto en el PIB sería igual.

\section{OPINIONES DE EXPERTOS, REALES E IMAGINADAS}

Nordhaus hizo las dos únicas encuestas de "opiniones de expertos" para estimar el impacto del calentamiento global sobre el PIB, en 1994 (Nordhaus, 1994a) y 2017 (Nordhaus y Moffat, 2017). En la primera pidió a personas de diversos antecedentes académicos que dieran sus estimaciones del impacto de tres escenarios de calentamiento global en el PIB: a) un aumento de $3{ }^{\circ} \mathrm{C}$ para 2090; b) un aumento de $6{ }^{\circ} \mathrm{C}$ para 2175; y (C) un aumento de $6{ }^{\circ} \mathrm{C}$ para 2090. Los números de este estudio utilizados por el IPCC (gráfica 1) fueron un aumento de temperatura de $3{ }^{\circ} \mathrm{C}$ para una caída del PIB del 3,6\%.

Las opiniones de los expertos son un procedimiento válido para agregar conocimiento en áreas que requieren la agregación de un gran número de campos dispares, como explicaron el científico climático Tim Lenton y sus coautores en "Elementos de inflexión en el sistema climático de la Tierra" (Lenton et al., 2008):

La solicitud formal de las opiniones de expertos se suele utilizar para aportar a la comprensión actual de los estudios de modelos, de la evidencia empírica y de las consideraciones teóricas para influir en variables relevantes de política. Desde la perspectiva de las ciencias naturales, una crítica general es que las opiniones de expertos tienen sesgos subjetivos y, además, que no se suman al cuerpo de conocimiento científico salvo que sean verificadas con datos o teoría. No obstante, las consultas a expertos, basadas en rigurosos protocolos de la estadística y el análisis de riesgo, han demostrado ser una valiosa fuente de información en la formulación de políticas públicas. Se reconoce cada vez más que también 
pueden cumplir un valioso papel para informar las decisiones de política climática (ibíd., p. 1791).

Aquí cito este artículo en contraste con el de Nordhaus por dos razones: 1) muestra cómo se deben hacer las encuestas de opinión a expertos; 2) Nordhaus después cita esta encuesta en apoyo de su uso de una "función de daño" del cambio climático que carece de puntos de inflexión, cuando la encuesta rechaza explícitamente tales funciones.

La encuesta de Lenton et al. se envió a 193 científicos, y 52 de ellos la respondieron. A los encuestados se les pidió que se ciñeran a su área de conocimiento, en vez de especular de manera más amplia: "Se alentó a los participantes a mantenerse en su área de especialización” (Lenton et al., 2008, p. 10), como muestra el cuadro 2.

Cuadro 2

Campos de especialización de los expertos encuestados

\begin{tabular}{lc}
\hline \multicolumn{1}{c}{ Campo } & Número \\
\hline Glaciología & 10 \\
Modelación de la capa de hielo & 3 \\
Ecología & 4 \\
Modelación de ecosistemas & 7 \\
Modelación de la biosfera marina & 4 \\
Oceanografía & 9 \\
Modelación del clima & 15 \\
\hline
\end{tabular}

Fuente: resumen de la tabla 1 de Lenton et al. (2008, p. 10).

La encuesta de Nordhaus empezó con una carta que solicitaba la participación a 22 personas; 18 de ellas cumplieron plenamente y una parcialmente. Nordhaus las describe así: 10 economistas, 4 "otros científicos sociales" y 5 "científicos naturales e ingenieros"; también señala que 8 de los economistas provienen de "otras subdisciplinas de la economía (cuyas principales preocupaciones se encuentran fuera de la economía ambiental)" (Nordhaus, 1994a, p. 48). Esto debería excluirlos ipso facto de participar en la encuesta a expertos.

Uno de ellos fue Larry Summers, quien quizá sea la fuente de las citas más selectas del artículo, tales como "En mi respuesta, el valor de existencia [de las especies] es irrelevante; no me importan las hormigas salvo para drogas" (ibíd., p.50).

La encuesta de Lenton combinó el conocimiento de sus entrevistados en campos específicos del cambio climático para compilar una lista de grandes elementos del sistema climático del planeta (> $1.000 \mathrm{~km}$ de extensión) que podían provocar un cambio cualitativo de estado por incrementos de la temperatura global de entre $0,5{ }^{\circ} \mathrm{C}$ (desaparición del hielo marino en el verano ártico) y $6{ }^{\circ} \mathrm{C}$ (un fenó- 
meno del Niño amplificado que causa sequías en el sudeste asiático y otros lugares), en escalas de tiempo que varían de 10 años (hielo marino en el verano ártico) a 300 años (desintegración de la plataforma de hielo de la Antártida Occidental) (Lenton et al., 2008, p. 1788).

La encuesta de Nordhaus se resumió en un par de números superficialmente anodinos -aumento de la temperatura de $3{ }^{\circ} \mathrm{C}$ y caída del PIB del 3,6\%-, pero ese resumen oculta mucho más de lo que revela. Hubo un amplio desacuerdo, bien documentado por Nordhaus, entre la relativamente pequeña cohorte de científicos reales encuestados y los economistas "cuyas principales preocupaciones se encuentran fuera de la economía ambiental”. Las citas de los economistas encuestados también revelan la fuente de la predisposición de los economistas en general a descartar la importancia del cambio climático.

Como señaló Nordhaus, "las estimaciones de los científicos naturales [de los daños del cambio climático] eran de 20 a 30 veces más altas que las de los economistas convencionales" (Nordhaus, 1994a, p. 49). La estimación promedio de los "economistas no ambientales" (ibíd., gráfica 4, p. 49) de los daños al PIB por un aumento de $3{ }^{\circ} \mathrm{C}$ para 2090 fue del 0,4\% del PIB; el promedio de los científicos naturales fue del 12,3\%, y uno de ellos se negó a responder las preguntas clave de Nordhaus:

Además, aunque la disposición de los encuestados a hacer estimaciones riesgosas de las probabilidades subjetivas fue alentadora, se debe subrayar que la mayoría de los encuestados presentaron estas estimaciones con reservas y reconociendo la dificultad inherente a la tarea. Un encuestado (19), sin embargo, se negó a hacer tales conjeturas y escribió:

Debo decir que me maravilla que los economistas estén dispuestos a hacer estimaciones cuantitativas de las consecuencias económicas del cambio climático cuando las únicas medidas disponibles son estimaciones de los incrementos promedio de la temperatura global en la superficie. Como [alguien] que ha pasado su carrera preocupándose por los caprichos de la dinámica de la atmósfera, me maravilla que puedan traducir un solo número global, un sustituto muy malo de una descripción de las condiciones climáticas, a estimaciones cuantitativas de los impactos globales en las condiciones económicas (ibíd., pp.50-51).

Los comentarios de los economistas están en el otro extremo del espectro de los de este científico. Como creían firmemente en la capacidad de adaptación de las "sociedades humanas", nacida de su aceptación del modelo neoclásico del capitalismo, en el que "la economía" siempre vuelve al equilibrio después de un "choque exógeno", no podían imaginar que el cambio climático pueda causar un daño significativo a la economía, sea lo que sea que haga a la biosfera: 
Un encuestado sugirió caprichosamente que no era sorprendente, pues los economistas saben poco acerca de la intrincada red de ecosistemas naturales, mientras que los cientificos naturales saben igualmente poco acerca de la increible adaptabilidad de las sociedades humanas [...]

Hay una clara diferencia de perspectiva entre los encuestados, dependiendo de sus supuestos sobre la capacidad de la sociedad para adaptarse a cambios climáticos. A uno le preocupaba que la respuesta de la sociedad en el próximo milenio fuera similar a la prevalente en la Edad Media, mientras que otro dijo que el grado de adaptabilidad de las economias humanas es tan alto que para la mayoría de los escenarios el impacto del calentamiento global sería "en esencia cero".

Un economista explicó que, en su opinión, la energía y la capacidad intelectual son los únicos límites para el crecimiento en el largo plazo, y con cantidades suficientes de ellas es posible adaptar o desarrollar nuevas tecnologías para evitar costos económicos significativos (ibíd., pp. 48-49; todas las cursivas son añadidas).

Dada esta extrema divergencia de opiniones entre economistas y científicos, se podría imaginar que la próxima encuesta de Nordhaus examinaría las razones para ello. De hecho, hizo lo contrario: su metodología excluyó por completo a los no economistas.

En vez de una encuesta a expertos, fue una revisión de literatura (Nordhaus y Moffat, 2017), que ipso facto es otro método legítimo para obtener datos sobre un tema difícil de medir y sujeto a gran incertidumbre. Él y su coautor buscaron artículos relevantes utilizando la cadena “(daño O impacto) Y clima Y costo” (ibíd., p. 7), lo que es razonable, aunque demasiado amplio (como admiten en su artículo).

La falla clave en esta búsqueda fue dónde buscaron: ejecutaron su cadena de búsqueda en Google, que arrojó 64 millones de resultados, en Google Scholar, que arrojó 2,8 millones, y en la base de datos Econlit, que solo arrojó 1.700 estudios. Como había demasiados resultados en Google y Google Scholar ignoraron esos resultados y simplemente revisaron los 1.700 artículos de Econlit (ibíd., 2017, p. 7). Articulos escritos casi exclusivamente por economistas.

Nordhaus y Moffat leyeron los 1.700 resúmenes para excluir casi todos los artículos, excepto 24. La lectura de estos artículos llevó a que solo incluyeran 11 en los resultados. Complementaron esta "síntesis de investigación sistemática (SRS)" con:

un segundo enfoque, conocido como "resumen de investigación no sistemática". En este enfoque, el universo de estudios se seleccionó combinando métodos formales e informales, como la SRS anterior, los resultados de la encuesta Tol y otros estudios conocidos por los investigadores (ibíd., p. 8). 
Su labor llevó a la adición de solo cinco estudios que no fueron utilizados por el IPCC ni por Tol en sus artículos de agregación (Tol, 2009, 2018), con 6 resultados adicionales y 4 autores más -Cline, Dellink, Kemfert y Hambel- que aún no habían citado en la literatura sobre estimaciones empíricas (aunque Cline fue uno de los entrevistados por Nordhaus en su encuesta de 1994).

Sorprendentemente, dado que Nordhaus fue el autor principal de este estudio, uno de los estudios no utilizados anteriormente fue Nordhaus (2010). (Nordhaus y Moffat (2017) no dan detalles de ese artículo, ni de ningún otro que hayan descubierto, pero presumo que es Nordhaus (2010), por la fecha y porque la temperatura y las estimaciones de daños que allí aparecen -un aumento de la temperatura de $3,4{ }^{\circ} \mathrm{C}$ que ocasiona una caída del PIB del 2,8\%- son idénticas a las que se presentan en el cuadro 2 de este escrito.

Puede parecer extraño que Nordhaus no advirtiera que su artículo no se incluía en estudios anteriores. Pero hay una buena razón para esta omisión: Nordhaus (2010) no fue un estudio enumerativo ni estadístico, y mucho menos resultado de una "solicitud a expertos", ;sino el resultado de correr el "Modelo de evaluación integrada" (MEI) del

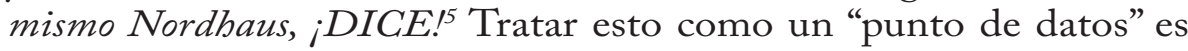
usar el resultado de un modelo para calibrar el mismo modelo ${ }^{6}$. No obstante, estos números $-\mathrm{y}$ los cinco pares adicionales de los cuatro estudios adicionales descubiertos en su búsqueda- se añadieron a la lista de números a partir de los cuales economistas como Nordhaus calibran lo que llaman sus "funciones de daño".

\section{FUNCIONES DE DAÑO}

Las “funciones de daño" son el medio que Nordhaus y muchos otros economistas neoclásicos usan para conectar las estimaciones de los científicos sobre el cambio de la temperatura global con las de ellos mismos, como se muestra en secciones anteriores, que son estimaciones totalmente erróneas del PIB futuro dado este cambio de temperatura. Reducen el PIB de lo que pretenden que habría sido en ausencia total de cambio climático a lo que dicen que será dados diferentes niveles de aumento de la temperatura. La forma que adoptan estas funciones de daño suele ser simplemente cuadrática:

${ }^{5}$ DICE significa clima y economía dinámicos e integrados.

${ }^{6}$ Por esta misma razón, no considero el uso de modelos de Equilibrio General Computable para generar números y calibrar el MEI, la cuarta técnica listada por el IPCC en Arent et al. (2014, p. SM10-4). 
Nordhaus justifica el uso de una función cuadrática para describir un proceso tan intrínsecamente discontinuo como el cambio climático malinterpretando la literatura cientifica, en particular el cuidadoso estudio de las opiniones de expertos realizado por Lenton et al. (2008) y antes contrastado con la revisión de Nordhaus (1994a) de las opiniones de una mayoría de no expertos. Nordhaus hace la siguiente declaración en su manual DICE, y la repite en Nordhaus y Moffat (2017, p. 35):

La versión actual supone que los daños son una función cuadrática del cambio de temperatura y no incluye umbrales nítidos ni puntos de inflexión, pero esto es consistente con la encuesta de Lenton et al. (2008) (Nordhaus y Sztorc, 2013, p. 11; cursivas añadidas).

En The climate casino, Nordhaus afirma:

Se han hecho algunos estudios sistemáticos de los puntos de inflexión en los sistemas terrestres. Uno particularmente interesante, de Lenton y sus colegas, examinó los elementos de inflexión importantes y evaluó su temporalidad [...] Su revisión no encuentra elementos de inflexión criticos en un horizonte temporal de menos de 300 años hasta que las temperaturas globales hayan aumentado en al menos $3{ }^{\circ} \mathrm{C}$ (Nordhaus, 2013, p. 60, cursivas añadidas).

Estas afirmaciones son tergiversaciones flagrantes de "Elementos de inflexión en el sistema climático de la Tierra" (Lenton et al., 2008). El primer elemento en la tabla de resultados de Lenton et al. cumple los dos criterios numéricos que mencionó Nordhaus: el hielo marino del verano ártico podría ser provocado por un calentamiento global de entre 0,5 y $2{ }^{\circ} \mathrm{C}$, y en un lapso de tiempo medido en décadas; ver la gráfica 3 .

Gráfica 3

Extracto de la Tabla 1 de Lenton et al.

\begin{tabular}{|c|c|c|c|c|c|c|}
\hline Tipping element & $\begin{array}{l}\text { Feature of } \\
\text { system, } F \\
\text { (direction of } \\
\text { change) }\end{array}$ & $\begin{array}{c}\text { Control } \\
\text { parameter(s), } \rho\end{array}$ & $\begin{array}{c}\text { Critical } \\
\text { value }(s),{ }^{\dagger} \rho_{\text {crit }}\end{array}$ & $\begin{array}{c}\text { Global } \\
\text { warming }\end{array}$ & $\begin{array}{c}\text { Transition } \\
\text { timescale, }{ }^{\dagger} T\end{array}$ & Key impacts \\
\hline Arctic summer sea-ice & Areal extent (-) & $\begin{array}{l}\text { Local } \Delta T_{\text {air }} \text {, ocean heat } \\
\text { transport }\end{array}$ & Unidentified 5 & $+0.5-2^{\circ} \mathrm{C}$ & $\approx 10 \mathrm{yr}$ (rapid) & $\begin{array}{l}\text { Amplified warming, } \\
\text { ecosystem change }\end{array}$ \\
\hline
\end{tabular}

Fuente: Lenton et al. (2008, Tabla 1, p. 1788).

Nordhaus justifica su omisión mediante un tercer criterio de "nivel de preocupación" que aparece en su tabla N1, donde el hielo marino del 
verano ártico recibe la clasificación más baja $\left(^{*}\right)$. En apariencia, esto justifica su afirmación de que "no habría ningún punto de inflexión crítico" en menos de 300 años, y con un aumento de temperatura de menos de $3{ }^{\circ} \mathrm{C}$.

Gráfica 4

Tabla de Nordhaus que pretende resumir los resultados de Lenton

Table N-1.

\begin{tabular}{|c|c|c|c|c|}
\hline Tipping element & $\begin{array}{c}\text { Time scale } \\
\text { (years) }\end{array}$ & $\begin{array}{l}\text { Threshold } \\
\text { warming } \\
\text { value }\end{array}$ & $\begin{array}{l}\text { Level of concern } \\
\text { (most concern } \\
=* * *)\end{array}$ & Concern \\
\hline $\begin{array}{l}\text { Arctic summer } \\
\text { sea ice }\end{array}$ & 10 & $+0.5-2^{\circ} \mathrm{C}$ & * & $\begin{array}{l}\text { Amplified } \\
\text { warming, ecosystems }\end{array}$ \\
\hline $\begin{array}{l}\text { Sahara/Sahel } \\
\text { and West } \\
\text { African } \\
\text { monsoon }\end{array}$ & 10 & $+3-5^{\circ} \mathrm{C}$ & $* *$ & Wet period \\
\hline
\end{tabular}

Fuente: (Nordhaus, 2013, p. 333).

Pero esa columna no existe en la Tabla 1 de Lenton et al. (2008) ${ }^{7}$, mientras que su análisis de la clasificación de las amenazas sitúa el hielo marino del verano ártico en el primer lugar y no en el último:

Concluimos que la mayor ( $y$ más clara) amenaza es para el Ártico, donde es probable que la pérdida de hielo marino en verano ocurra mucho antes (y contribuya potencialmente al) derretimiento de la CHG (Lenton et al., 2008, pp. 1791-92, cursivas añadidas).

Su tratamiento del tiempo también difiere sustancialmente del que da a entender Nordhaus, quien sostiene que las decisiones sobre los elementos de inflexión con horizontes de tiempo de varios siglos pueden dejarse a quienes tomen decisiones dentro de algunos siglos. Aunque Lenton et al. dan un plazo mayor de 300 años para el derretimiento total de la capa de hielo de Groenlandia ( $\mathrm{CHG})$, por

7 La columna "Valores críticos" de la Tabla 1 de Lenton et al. se refiere a si existe una magnitud empírica conocida que desencadene el punto de inflexión, no a si el punto de inflexión en sí es de importancia crítica. El símbolo junto a la expresión "No identificado", que se utiliza para describir el hielo marino del verano ártico, indica que "El significado de la teoría, los resultados del modelo o los paleo-datos sugieren la existencia de un umbral crítico, pero la literatura carece de un valor numérico" (Lenton, et al. 2008, p. 1788). 
ejemplo; señalan que solo consideraron elementos de inflexión cuyo destino debe decidirse en este siglo:

Nos centramos, entonces, en las consecuencias de las decisiones tomadas en este siglo que desencadenan un cambio cualitativo en este milenio, y excluimos los elementos de inflexión cuyo destino se decida después de 2100 (ibíd., p. 1787).

Por tanto, si bien la CHG podría no derretirse totalmente en varios siglos, las acciones humanas que decidirán si eso sucede o no se tomarán en este siglo, no en varios cientos de años a partir de hoy.

Por último, la conclusión del artículo comenzó advirtiendo que no se deben utilizar funciones suaves y señaló que es probable que se activen puntos de inflexión climáticos discontinuos en este siglo; además, reiteró que las mayores amenazas eran el hielo marino del verano ártico y de Groenlandia:

\section{Conclusión}

La sociedad puede dejarse llevar por una falsa sensación de seguridad mediante proyecciones suaves del cambio global. Nuestra síntesis del conocimiento actual indica que diversos elementos de inflexión podrían alcanzar su punto crítico en este siglo, por el cambio climático antropogénico. Las mayores amenazas son la inflexión del hielo marino del Ártico y de la capa de bielo de Groenlandia, y al menos otros cinco elementos podrían sorprendernos presentando un punto de inflexión cercano ((ibíd., 2008, p. 1792; cursivas añadidas).

Pregunté a Lenton si había alguna razón para la interpretación de Nordhaus de su artículo que yo hubiera pasado por alto (Keen y Lenton, 2020). Respondió que no, que mi interpretación del artículo era correcta y que había otros artículos que también rechazaban rotundamente la proposición de que una función suave es adecuada para evaluar los peligros del cambio climático (Cai et al., 2016; Kriegler et al., 2009; Lenton y Ciscar, 2013; Lenton et al., 2019).

No hay entonces ninguna justificación empírica ni científica para elegir una función cuadrática para representar los daños del cambio climático; de hecho, se aplica una función contraria. Pese a ello, esa es la función que adoptó Nordhaus. Suponiendo dicha forma funcional, las únicas incógnitas son los valores de los coeficientes $a, b$ y $c$ de la ecuación (2).

\section{¿CUAN BAJO SE PUEDE IR?}

Desde que Nordhaus empezó a usar una función cuadrática, él ha reducido continuamente el valor de sus parámetros, de un 0,0035 
inicial para el término cuadrático -lo que significa que se supone que el calentamiento global reduce el PIB en un 0,35\% por la temperatura (cambio sobre los niveles preindustriales) al cuadrado hasta un valor final de 0,00227 [ver la ecuación (3)]. Los documentos fuente son aquí: Nordhaus y Sztorc (2013, pp. 83, 86, 91 y 97) para las versiones DICE de 1992, 1999, 2008 y 2013), Nordhaus (2017, p. 1) para la de 2017 y Nordhaus (2018b, p. 345) para la de 2018):

\begin{tabular}{|c|c|c|}
\hline Año & Función de daño & Parámetros \\
\hline & $\frac{1}{1+\frac{a}{9} \times \sqrt{T}}$ & $a=0.0133$ \\
\hline \multirow{2}{*}{999} & 1 & $b=0.0045$ \\
\hline & $\begin{array}{c}1+b \times T+c \times T^{2} \\
1\end{array}$ & $c=0.0035000$ \\
\hline 2008 & $\frac{1}{1+c \times T^{2}}$ & $c=0.0028388$ \\
\hline & $\overline{1+c \times T^{2}}$ & $c=0.0026700$ \\
\hline 17 & $1-c \times T^{2}$ & $c=0.0023600$ \\
\hline 18 & $1-c \times T^{2}$ & $c=0.0022700$ \\
\hline
\end{tabular}

Esta disminución redujo progresivamente sus ya triviales predicciones del daño del calentamiento global al PIB. Por ejemplo, su predicción del impacto sobre el PIB de un aumento de la temperatura de $4{ }^{\circ} \mathrm{C}$ -el nivel que considera óptimo en su conferencia Nobel, pues según su modelo minimiza los costos conjuntos del daño y el abatimiento (Nordhaus, 2018a, diapositivas 6 y 7)- se redujo de una caída del 7\% en 1992 a una caída del 3,6\% en 2018 (ver la gráfica 5).

Paso ahora a hacer lo que el mismo Nordhaus dijo que debería hacer un científico cuando ridiculizó el modelo de Forrester: "requerir la validación empírica de los supuestos o las predicciones del modelo antes de declarar su contenido de verdad" (Nordhaus, 1973, p. 1183). Esto es claramente algo que ni Nordhaus ni ningún otro economista neoclásico del cambio climático hicieron, aparte de las menciones honoríficas antes señaladas.

\section{DESMONTE DE LOS DELIRIOS NEOCLÁSICOS: PIB Y ENERGÍA}

Nordhaus justificó el supuesto de que el 87\% del PIB no será afectado por el cambio climático con base en que:

para la mayor parte de la economía - manufactura, minería, servicios públicos, finanzas, comercio y la mayoría de las industrias de servicios- es difícil encontrar grandes impactos directos del cambio climático proyectado durante los próximos 50 a 75 años (Nordhaus, 1991, p. 932). 
En realidad, se puede identificar fácilmente superando la falla de los economistas en general -no solo de los neoclásicos- para apreciar el papel de la energía en la producción. Casi todos los modelos económicos utilizan funciones de producción que suponen que el "trabajo" y el "capital" son todo lo que se necesita para hacer el "producto". Pero ni el trabajo ni el capital pueden funcionar sin insumos de energía: "para acuñar una frase, el trabajo sin energía es un cadáver, mientras que el capital sin energía es una escultura" (Keen et al., 2019, p. 41). La energía se necesita directamente para producir el PIB; por tanto, si la producción de energía tiene que caer debido al calentamiento global, también caerá el PIB.

Gráfica 5 ¿Qué tan bajo se puede llegar?

Revisiones a la baja de Nordhaus a su función de daño, 1992-2018

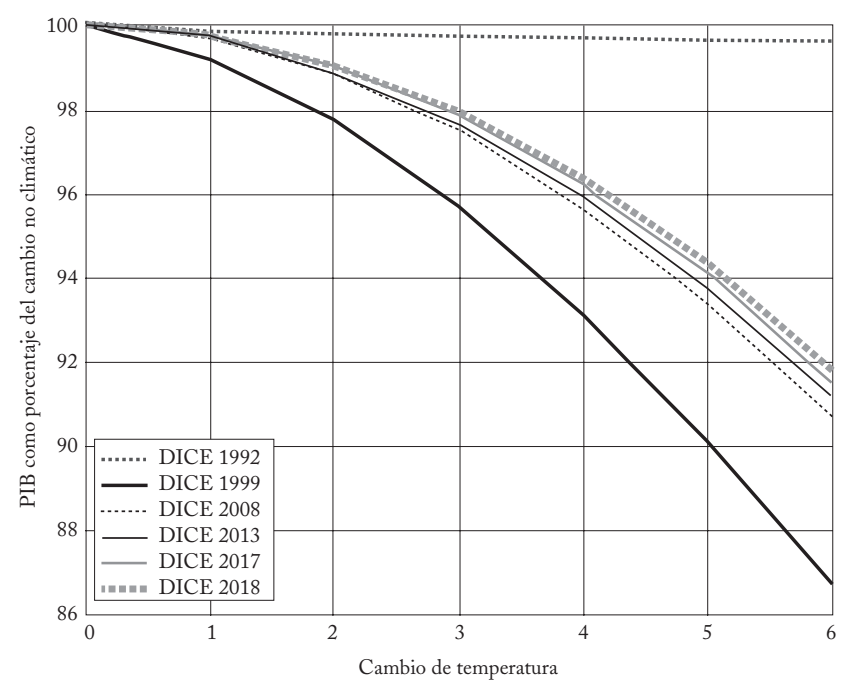

Fuentes: DICE 1992,1999, 2008 y 2013: Nordhaus y Sztorc (2013); DICE 2017: Nordhaus (2017) y DICE 2018: Nordhaus (2018b).

La única pregunta es cuánto, y la respuesta, dada nuestra dependencia de los combustibles fósiles: mucho. A diferencia de la correlación trivial entre temperatura local y PIB local utilizada por Nordhaus y sus colegas en el método "estadístico", la correlación entre producción global de energía y PIB global es muy fuerte. Una regresión lineal simple entre producción de energía y PIB da un coeficiente de correlación de 0,997; ver la gráfica $6^{8}$.

${ }^{8}$ Aquí utilizo una regresión lineal tosca para enfatizar cuán incorrecto es que los neoclásicos dejen de lado el impacto de la energía cuando hablan del cambio climático. Una regresión log-log, que es más adecuada para la 
Gráfica 6

La energía determina el PIB

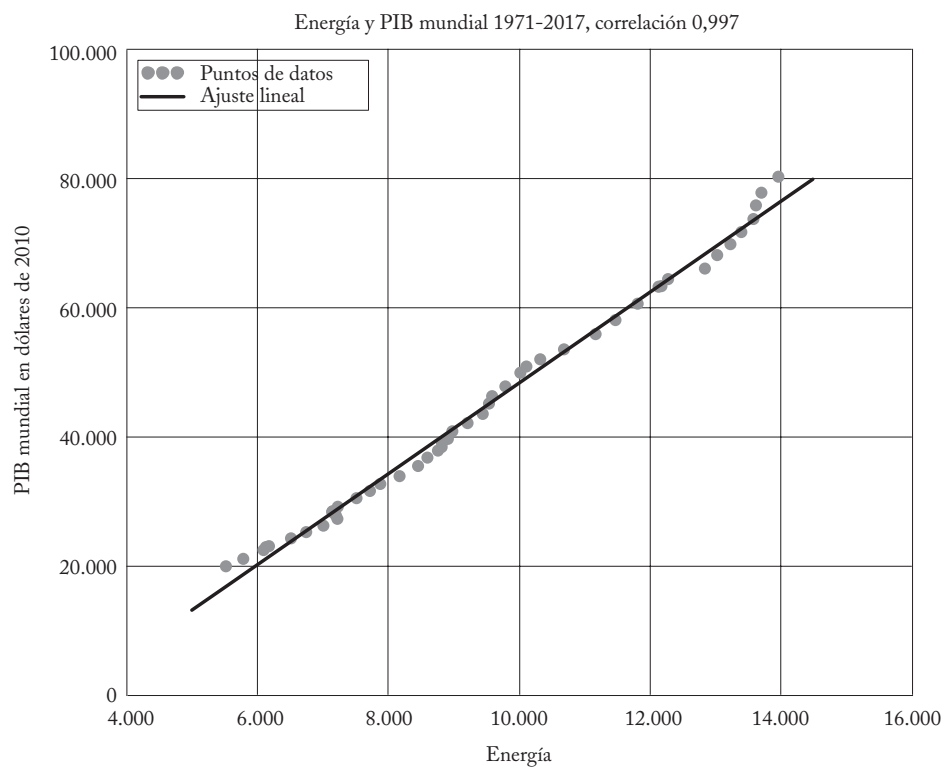

Fuentes: PIB: https: [data.worldbank.org/indicator/NY.GDP.MKTP.KD]; Energía: https: [data.oecd.org/ nergy/primary-energy-supply.htm\#indicator-chart].

A su vez, el PIB determina el exceso de $\mathrm{CO}^{2}$ en la atmósfera. Una regresión lineal entre PIB y $\mathrm{CO}^{2}$ arroja un coeficiente de correlación de 0,998; ver la gráfica 7 .

Por último, el $\mathrm{CO}^{2}$ determina fuertemente el exceso de temperatura sobre los niveles preindustriales. Una regresión lineal entre el $\mathrm{CO}^{2}$ y la anomalía de la temperatura global da una correlación de 0,992 usando datos suavizados (que excluyen el efecto de fluctuaciones distintas del $\mathrm{CO}^{2}$, como el efecto de E1 Niño) (gráfica 8)9

Trabajando a la inversa, si los cambios climáticos causados por el aumento de la temperatura global persuaden al público y a los diseñadores de política de que debemos dejar de añadir $\mathrm{CO}^{2}$ a la atmósfera "hoy", sea cuando sea "hoy", el PIB mundial caerá en forma aproximadamente proporcional a la relación entre producción de energía de combustibles fósiles y producción total de energía en ese momento.

Ya en 2020, los combustibles fósiles aportaban un 85\% de la producción de energía. De modo que si 2020 fuese el año en que la extrapolación hacia adelante o hacia atrás de esta relación, tiene un coeficiente de correlación aún mayor: 0,998. Se debe utilizar una relación no lineal adecuada en todo modelo realista del cambio a largo plazo.

${ }^{9}$ La correlación con datos no suavizados sigue siendo sumamente alta: 0,958. 


\section{Gráfica 7}

Sin una descarbonización significativa, el PIB determina el $\mathrm{CO}^{2}$

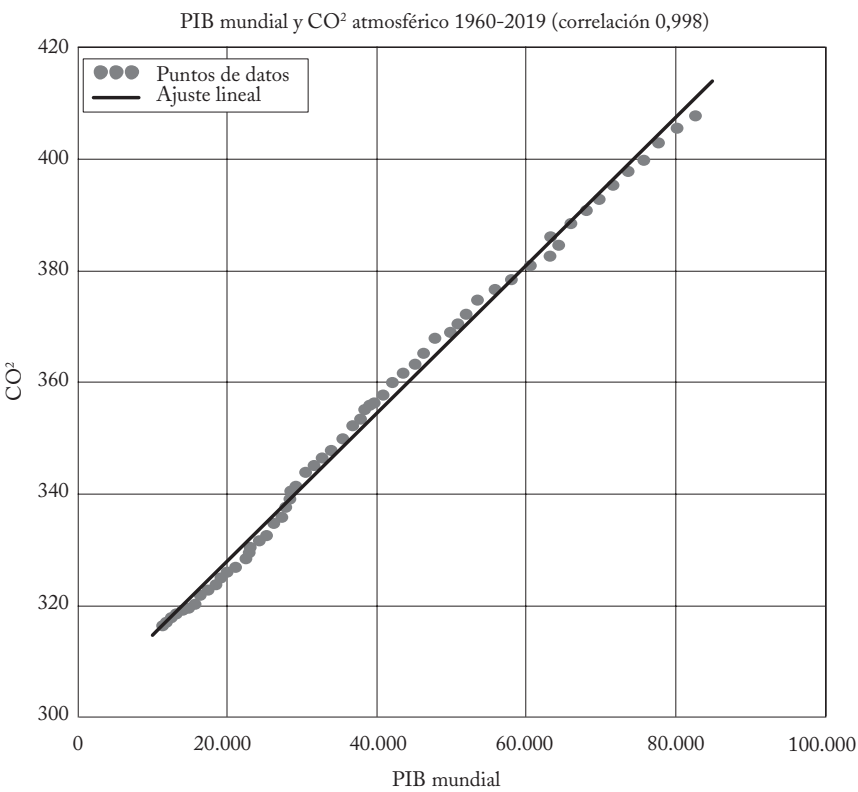

Fuente: PIB mundial dólares de 2010: [https://data.worldbank.org/Indicator/NY.GDP.MKTP.KD]; CO²: https: [scripsco2.ucsd.edu/data/atmospheric_co2/primary_mlo_co2_record.html].

\section{Gráfica 8}

$\mathrm{E} 1 \mathrm{CO}^{2}$ determina el calentamiento global

$\mathrm{CO}^{2}$ atmosférico y anomalía de temperatura global 1958-2020 (correlación 0,992)

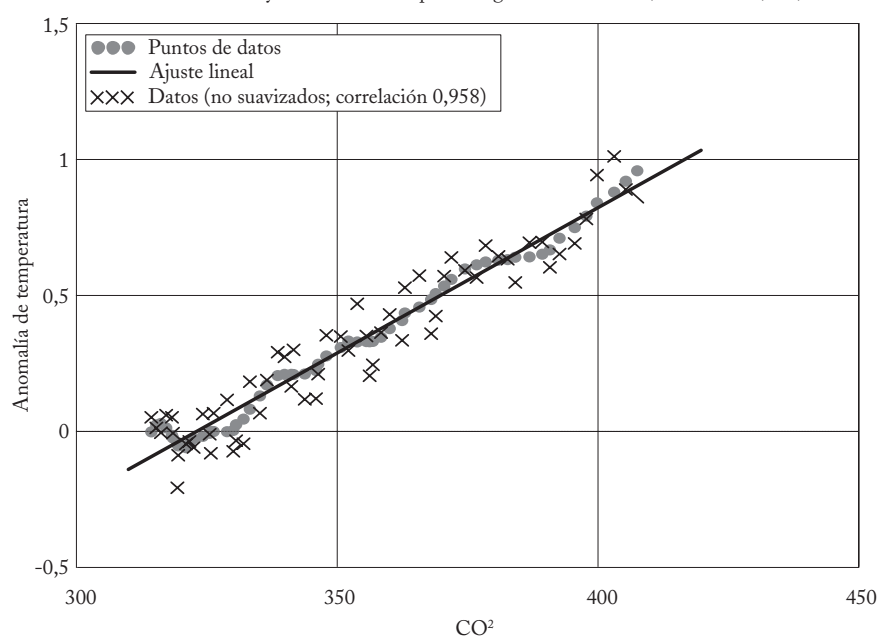

Fuentes: Anomalía de temperatura: [https: data.giss.nasa.gov/gistemp/graphs_v4/] CO²: [https://scripsco2. ucsd.edu/data/atmospheric_co2/primary_mlo_co2_record.html]. 
humanidad decide detener el crecimiento del $\mathrm{CO}^{2}$, el PIB caería en un orden del $85 \%$. Aun si se mantuviese la muy alta tasa de crecimiento de las energías renovables de 2015 -cuando la relación entre energías renovables y producción total de energía aumentó cerca de un 3\% anual-, las energías renovables seguirían produciendo menos del 40\% de la energía total en 2050 (gráfica 9). Esto implica una caída del PIB de un $60 \%$ en ese momento. Solo sobre esta base, la decisión de los economistas neoclásicos del cambio climático de excluir "la manufactura, la minería, los servicios públicos, las finanzas, el comercio y la mayoría de las industrias de servicios" de cualquier consecuencia del cambio climático es totalmente injustificada.

Gráfica 9

Energía renovable como porcentaje de la producción total de energía

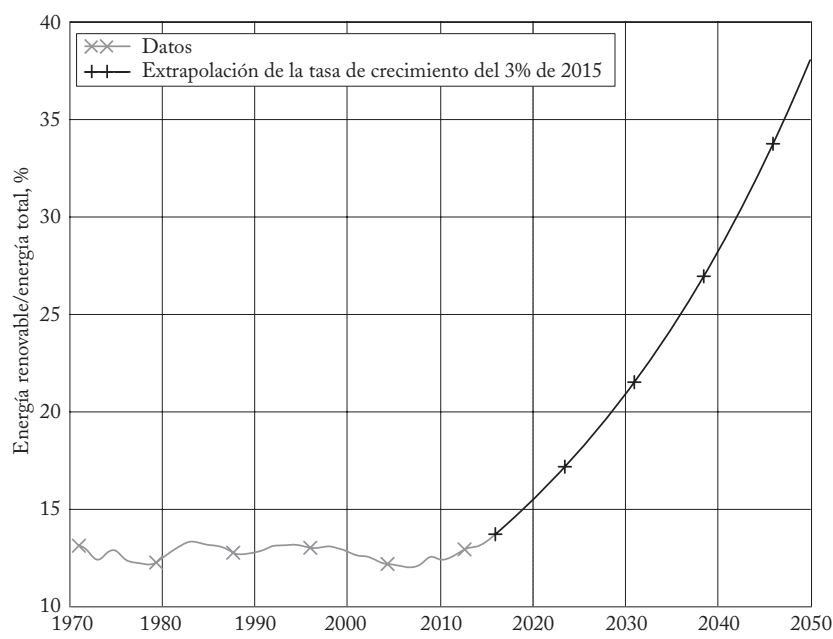

\section{DESMONTE DE LOS DELIRIOS NEOCLÁSICOS: ESTADÍSTICA}

El "enfoque de corte transversal", que usa los coeficientes de la relación entre temperatura geográfica y PIB como proxy de la relación entre temperatura y PIB globales es igualmente injustificado. Supone que no importa cómo se altere la temperatura: el efecto sobre el PIB será igual. Tol defendió esta opinión en un intercambio en Twitter conmigo, el científico del clima Daniel Swain (Swain et al., 2020) y el profesor de Astrofísica Computacional Ken Rice (Köhler et al., 2018) del 17-18 de junio de $2019^{10}$ :

${ }^{10}$ Este y un intercambio por Twitter posterior que se cita en este artículo fueron ligeramente editados para mejorar el tono y corregir errores ortográficos. 
Richard Tol: $10 \mathrm{~K}$ es menor que la distancia de temperatura entre Alaska y Maryland (casi igualmente ricos), o entre Iowa y Florida (casi igualmente ricos). El clima no es un determinante principal del ingreso [https:// twitter.com/RichardTol/status/1140591420144869381?s=20].

Daniel Swain: Un clima global 10 grados más cálido que el actual no es remotamente lo mismo que tomar el clima actual y añadir 10 grados en todas partes. Ese es un concepto erróneo muy difundido, pero demasiado peligroso [https://twitter.com/Weather_West/status/114067064 7313584129 ? $=20]$.

Richard Tol: Ese no es el punto, Daniel. Observamos que las personas prosperan en climas muy diferentes, y que algunas prosperan y otras no en el mismo clima. El determinismo climático no tiene entonces respaldo empírico [https://twitter.com/RichardTol/status/1140928458853421057?s=20].

Richard Tol: Y si una relación no es válida para las variaciones climáticas en el espacio, no se puede afirmar con seguridad que se mantenga en el tiempo [https://twitter.com/RichardTol/status/1140928893878263808?s=20].

Steve Keen: La causa de las variaciones en el espacio es totalmente diferente de las del tiempo. Que son comparables es el "supuesto simplificador" más ridículo y peligroso en la historia de la economía [https:// twitter.com/ProfSteveKeen/status/1140941982082244608?s=20].

Ken Rice: ¿Puedo aclararlo? ¿Sugiere realmente que sería manejable un aumento de $10 \mathrm{~K}$ en la temperatura superficial promedio global? [https:// twitter.com/theresphysics/status/1140661721633308673?s=20].

Richard Tol: Nos mudaríamos puertas adentro, como los saudíes [https:// twitter.com/RichardTol/status/1140669525081415680?s=20].

Igual que la decisión de excluir cerca del 90\% del PIB de los daños del cambio climático, la equivalencia supuesta por Tol entre cambios climáticos en el espacio y cambio climático en el tiempo ignora el papel de la energía como causa del cambio climático. Esto se puede ilustrar glosando su tercer trino sobre la cantidad de energía necesaria para provocar un incremento de $10{ }^{\circ} \mathrm{C}$ en la temperatura de la atmósfera:

Y si una relación no es válida para las variaciones climáticas en el espacio [sin cambiar el nivel de energía de la atmósfera], no se puede afirmar con seguridad que se mantenga en el tiempo [ya que la energía solar retenida en la atmósfera aumenta en más de 50.000 millones de Terajulios] (Trenberth, 1981).

Para expresar este nivel de energía en términos más comprensibles, equivale a 860 millones de bombas atómicas de Hiroshima, o 1,6 bombas por kilómetro cuadrado de la superficie del planeta ${ }^{11}$.

11 Trenberth estima la masa de la atmósfera en $513,7 \times 10^{18} \mathrm{~kg}$ (1981, p. 5238). Elevar la temperatura de un $\mathrm{kg}$ de aire en $1{ }^{\circ} \mathrm{C}$ requiere 1.004 julios de energía: el producto es $5.158 \times 1022$ julios, o 51.575 millones de Terajulios. 
Un aumento de la temperatura promedio de $10{ }^{\circ} \mathrm{C}$ también llevaría a temperaturas de "bulbo húmedo" sostenidas que serían fatales para los humanos en los trópicos y gran parte de los subtrópicos (Raymond et al., 2020; Xu et al., 2020). Es del orden del aumento que causó la extinción del final del Pérmico, la extinción masiva más extrema en la historia de la Tierra (Penn et al., 2018). Y cinco veces el nivel del incremento de la temperatura global $\left(2{ }^{\circ} \mathrm{C}\right)$ que los científicos del clima temen que pueda desencadenar "disparos de cascadas" que podrían transformar la Tierra en un "planeta invernadero" (Lenton et al., 2019; Steffen et al., 2018) potencialmente incompatible con la existencia humana:

Es probable que la Tierra invernadero sea incontrolable y peligrosa para muchos, en particular si hacemos la transición en solo uno o dos siglos, y plantea graves riesgos para la salud, las economías, la estabilidad política (es especial para los más vulnerables al clima) y, en última instancia, la habitabilidad del planeta para los humanos (Steffen et al., 2018, p. 8256).

Es entonces muy importante cómo se modifica la temperatura. A nivel planetario, hay tres determinantes principales de la temperatura media anual en cualquier punto del globo:

1. Las variaciones de la energía solar que llega a la Tierra;

2. Las variaciones de la cantidad de esta energía que retienen los gases de efecto invernadero; $y$

3. Las diferencias de ubicación en el planeta, principalmente las diferencias en la distancia al Ecuador.

Lo que hizo el "método de corte transversal" fue obtener parámetros para el tercer factor y luego suponer simplemente que esos mismos parámetros se aplicaban al segundo. Esto es comparable a medir cuidadosamente el terreno de una montaña en dirección norte-sur y luego usar esa información para asesorar sobre la seguridad de una expedición para atravesarla de oriente a occidente en la oscuridad.

\section{ECONOMETRÍA ANTES QUE ECOLOGÍA}

Esta debilidad del "enfoque de corte transversal" fue admitida en un artículo más reciente de esta tradición:

Una bomba de Hiroshima equivale a 60 Terajulios [https://www.justintools. com/unit-conversion/energy.php?k1=hiroshima-bomb-explosion\&k2=terajoules]. El área del planeta es de 510 millones de $\mathrm{km}^{2}$. Estos cálculos no tienen en cuenta la energía necesaria para elevar también la temperatura promedio de los océanos, como está haciendo el calentamiento global aunque más lentamente. Su masa es unas 250 veces la de la atmósfera. 
En primer lugar, la literatura se basa principalmente en el enfoque de corte transversal y, como tal, no tiene en cuenta la dimensión temporal de los datos (es decir, supone que la relación observada entre países también se mantiene en el tiempo) (Kahn et al., 2019, p. 2; cursivas añadidas).

Desafortunadamente, este comienzo prometedor fue neutralizado por su eventual extrapolación lineal simple del cambio en la relación temperatura y PIB entre 1960 y 2014 hasta 2100:

Empezamos documentando que la temperatura global promedio ha aumentado en $0,0181{ }^{\circ} \mathrm{C}$ por año en el último medio siglo [...] Mostramos que un aumento de la temperatura global promedio de $0,04{ }^{\circ} \mathrm{C}$ por año -correspondiente al escenario 8.5 de la senda representativa de concentración (RCP) [...] que supone mayor emisión de gases de efecto invernadero en ausencia de políticas de mitigación- reduce el PIB real per cápita mundial en un 7,22\% para 2100 (Kahn et al., 2019, p. 4).

Sus predicciones del cambio del PIB como función del cambio de temperatura corresponden a la región sombreada de la gráfica 10 (que reproduce su gráfica 2). La linealidad de su proyección es evidente: supone que no hay ningún cambio estructural en la relación entre temperatura global y PIB, incluso cuando la temperatura aumenta en $3,2^{\circ} \mathrm{C}$, en su horizonte temporal de 80 años $\left(0,04{ }^{\circ} \mathrm{C}\right.$ por año desde 2020 hasta 2100).

Gráfica 10

Extrapolación lineal de la relación temperatura-PIB, 1960-2014 a 2100

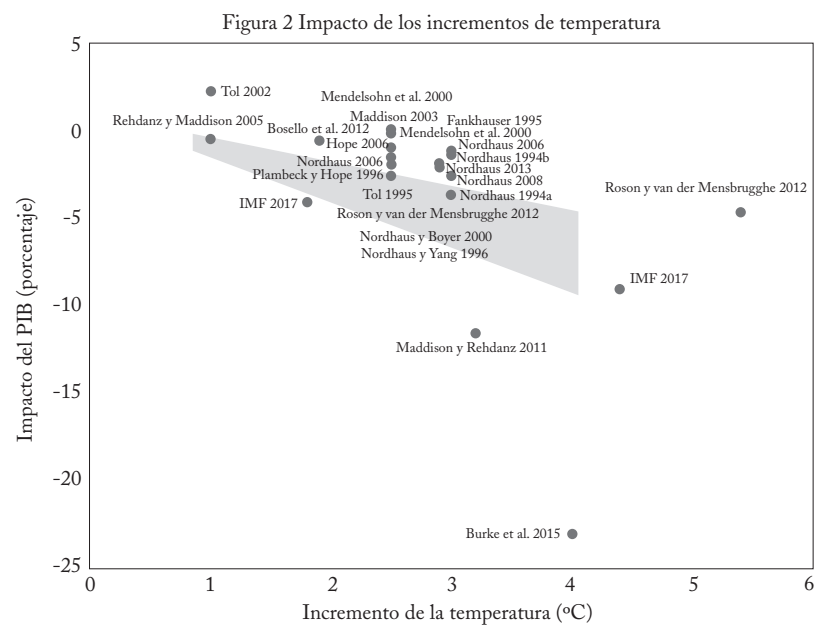

Fuente: (Kahn et al., 2019, p. 6 ) 
E1 31 de octubre de 2019, uno de los autores reconoció en Twitter la falla del artículo para explicar las obvias discontinuidades que tal aumento de temperatura provocará en el clima del planeta:

Kamiar Mohaddes: También quiero dejar en claro que no podemos afirmar, y no afirmamos, que nuestro análisis empírico permite eventos de desastres raros, bien sean tecnológicos o climáticos, lo que quizá sea una consideración importante. Desde esta perspectiva, los resultados contrafactuales que analizamos [...] en la sección 4 del artículo (ver https:// ideas.repec.org/p/cam/camdae/1965.html) se deben considerar conservadores porque solo tienen en cuenta escenarios donde los choques climáticos son gaussianos, sin permitir desastres raros [https://twitter.com/ KamiarMohaddes/status/1189846383307694084?s=20], [https://twitter. com/KamiarMohaddes/status/1189846648366796800?s=20].

Steve Keen: Kamiar, el punto en discusión en \#GlobalWarming es que cambia toda la distribución. Lo que es "raro" en nuestro clima actual como, p. ej., el derretimiento de Groenlandia- se convierte en una certeza a temperaturas más altas [https://twitter.com/ProfSteveKeen/status /1189849936290029569?s=20].

Lo que Mohaddes llamó "eventos de desastres raros", como la total desaparición de la capa de hielo ártico en el verano, sería en efecto raro a nuestra temperatura global actual. Pero se convierten en certezas cuando la temperatura sube otros $3{ }^{\circ} \mathrm{C}$ (Steffen et al., 2018, gráfica 3, p. 8255). Esta predicción es tan útil como un estudio de la relación entre temperatura y velocidad de patinaje que concluya que sería ventajoso aumentar la temperatura del hielo de $-2{ }^{\circ} \mathrm{C} \mathrm{a}+2{ }^{\circ} \mathrm{C}$.

Este artículo reciente me alertó sobre un estudio potencialmente prometedor que omití anteriormente: el significativo valor atípico de la gráfica 10 de Burke et al. (2015). Este estaba al menos fuera del estadio económico, si no en el de científicos como Steffen, que esperan que un incremento de la temperatura de $4{ }^{\circ} \mathrm{C}$ lleve al colapso de la civilización (Moses, 2020).

Como implica su título, "Efecto global no lineal de la temperatura sobre la producción económica”, al menos consideró no linealidades en el clima de la Tierra. Pero, de nuevo, se limitó a no linealidades en la relación entre 1960 y 2010, que luego se extrapolaron a un planeta futuro con un clima muy diferente:

Cuantificamos el impacto potencial del calentamiento en los ingresos nacional y global combinando nuestra función de respuesta no lineal estimada con escenarios "habituales" de calentamiento futuro y diferentes supuestos sobre el crecimiento económico y poblacional futuro de referencia. Este enfoque supone que las economias futuras responden a los cambios de temperatura de modo similar que las economias actuales; quizá un supuesto razonable dada la falta de adaptación observada en nuestra muestra 
de 50 años [...] el cambio climático reduce el producto global proyectado en un $23 \%$ en 2100 con respecto a un mundo sin cambio climático, aunque la incertidumbre estadística permite impactos positivos con una probabilidad de 0,29 (Burke et al., 2015, pp. 237-238; cursivas añadidas).

Como ocurre con gran parte de esa investigación, esos dos artículos muestran que los autores se deleitan con el éxtasis de la econometría, y que no ven la irrelevancia de su marco para el tema en cuestión.

\section{GIGO: ENTRA BASURA, SALE BASURA}

Cuando inicié este trabajo esperaba que la principal causa de las predicciones sumamente bajas de los daños por el cambio climático de Nordhaus sería la aplicación de una tasa de descuento muy alta (Nordhaus, 2007) ${ }^{12}$ a los daños climáticos estimados por científicos (Hickel, 2018), y que una crítica completa de su trabajo debería explicar por qué un modelo neoclásico basado en el equilibrio, como el DICE ${ }^{13}$, era una herramienta incorrecta para analizar algo tan incierto, dinámico y alejado del equilibrio como el cambio climático (Blatt, 1979; DeCanio, 2003) ${ }^{14}$. Descubrí, en cambio, que se aplicaba el adagio informático "Garbage in, garbage out"(GIGO): sin importar qué tan bueno o malo que sea el modelo real, cuando se alimenta con "datos" como los fabricados por Nordhaus y los economistas neoclásicos que lo siguieron. Las estimaciones numéricas a las que ajustaron sus inadecuados modelos, como se muestra aquí, no tienen ninguna relación con el calentamiento global. Incluso un modelo adecuado de la relación entre cambio climático y PIB arrojaría predicciones basura si se calibrara con "datos" como esos.

12 Nordhaus usa una tasa de descuento alta, y criticó a Stern por usar una mucho más baja. Pero la razón principal para que Nordhaus utilice una tasa alta es, como dijo, que "con la tasa de interés baja, los daños relativamente pequeños en los próximos dos siglos serán desbordados por los altos daños en los siglos y milenios que siguen al año 2200" (Nordhaus 2007, p. 202; cursivas añadidas). Como muestro aquí, la debilidad clave de su trabajo no es la tasa de descuento, sino la conclusión de que habrá "daños relativamente pequeños en los próximos dos siglos”.

${ }_{13}$ Aunque DICE significa clima y economía dinámicos e integrados, ese modelo es dinámico e integrado solo de nombre.

${ }^{14}$ DeCanio hace un muy buen trabajo sobre este tema, aunque su crítica se aplica igualmente bien a la aplicación de los modelos neoclásicos de "agente representativo" a cualquier tema macroeconómico, y no solo al cambio climático. Otras debilidades endémicas de este análisis incluyen la aplicación del análisis de costo-beneficio en vez del "Principio de precaución" a un tema tan incierto como el cambio climático, y el mal manejo de la incertidumbre por parte de la economía neoclásica en general. 
Esto suscita una pregunta clave: ¿cómo un trabajo tan claramente inadecuado logró la aprobación de los árbitros académicos?

\section{SUPUESTOS SIMPLIFICADORES Y REVISIÓN POR PARES: LOS DELANTEROS SE CONVIERTEN EN GUARDAMETAS}

Una de las razones por las que esa agenda de investigación no se ahogó al nacer fue la inclinación de los economistas neoclásicos a adoptar supuestos de los que dependen sus conclusiones, y luego rechazar toda objeción con base en que solo son "supuestos simplificadores".

Como observó Paul Romer, la justificación estándar para ello es "la afirmación metodológica de Milton Friedman, proveniente de una autoridad anónima, de que 'cuanto más significativa es la teoría, menos realistas son los supuestos"'(Romer, 2016, p. 5). Quienes recurren a esta defensa no parecen haber visto la nota de pie de página de Friedman: "La inversa de la proposición no se cumple, por supuesto: los supuestos que no son realistas (en este sentido) no garantizan una teoría significativa" (Friedman, 1953, p. 14).

Un supuesto simplificador es algo que, si se infringe, solo hace una pequeña diferencia en el análisis. Musgrave señala que "el supuesto de Galileo de que la resistencia del aire era insignificante, para los fenómenos que investigaba, era una afirmación verdadera sobre la realidad, y una parte importante de su explicación de esos fenómenos" (Musgrave, 1990, p. 380). Pero el tipo de supuestos que los economistas neoclásicos suelen adoptar es aquel en el que si el supuesto es falso la teoría no es válida (Keen, 2011, pp. 158-174).

Esto es claramente lo que sucede aquí con los supuestos centrales de Nordhaus y sus colegas neoclásicos. Si las actividades que ocurren puertas adentro están de hecho sujetas al cambio climático; si las relaciones entre temperatura y PIB en el espacio no se pueden utilizar como proxies del impacto del calentamiento global sobre el PIB, sus conclusiones son totalmente falsas. El cambio climático será al menos un orden de magnitud más perjudicial para la economía de lo que implican sus números, lo que solo puede llevar a rechazar su supuesto espúreo de que un $90 \%$ de la economía no será afectado por él. Puede ser mucho peor, muchísimo peor.

Desafortunadamente, es poco probable que los árbitros que aceptan el dictum de Friedman de que "una teoría no se puede probar por el 'realismo' de sus supuestos" (Friedman, 1953, p. 23) rechacen un artículo debido a sus supuestos, en especial si son los que aceptan los economistas neoclásicos. Así, las incursiones iniciales de Nordhaus en esta área recibieron un pase gratuito. 
Después, una debilidad del proceso de revisión por pares se hizo cargo. Como sabe cualquier académico publicado, una vez publica en un área, los editores de revistas lo nominarán como árbitro en esa área. Así, en vez de que la revisión por pares haga un control independiente de la veracidad de la investigación, puede facilitar la imposición de una hegemonía. Como fue uno de los primeros de los pocos economistas neoclásicos que trabajaron en el cambio climático, y el que se adelantó a presentar las primeras estimaciones empíricas de los daños del cambio climático a la economía, Nordhaus quedó en posición de enmarcar el debate y de jugar como guardameta. Cabe imaginar que disfrutó ese papel, no solo en vista de sus ataques a Forrester y a Los limites del crecimiento (Meadows et al., 1972; Nordhaus, 1973 y 1992), sino también de su ataque a su colega neoclásico, el economista $\mathrm{Ni}$ cholas Stern, por usar una tasa de descuento baja en The Stern Review (Nordhaus, 2007; Stern, 2007).

E1 resultado ha sido un grado indebido de conformidad en esta comunidad, que incluso Tol reconoció:

Es bastante posible que las estimaciones no sean independientes, pues solo hay un número relativamente pequeño de estudios, basados en datos similares, de autores que se conocen bien [...] aunque el número de investigadores que publicaron estimaciones del costo del daño marginal es mayor que el número de investigadores que publicaron estimaciones del impacto total, sigue siendo una comunidad relativamente pequeña y estrechamente unida, que puede estar sujeta al pensamiento de grupo, a la presión de sus pares y a la autocensura (Tol, 2009, pp. 37, 42-43).

En efecto; así es.

\section{CONCLUSIÓN: DRÁSTICA SUBESTIMACIÓN DE LOS DAÑOS ECONÓMICOS DEL CALENTAMIENTO GLOBAL}

Si el cambio climático fuese un área trivial de política pública, el pésimo trabajo que han hecho los economistas neoclásicos sobre el cambio climático no importaría mucho. Se podría tratar, como el engaño intencional de Sokal (2008), como un relato saludable de las debilidades de la academia.

Pero el impacto del cambio climático sobre la economía, la sociedad humana y la viabilidad de la biosfera de la Tierra en general son asuntos de suma importancia. Que se haya hecho un trabajo tan malo, y se lo haya tomado en serio, no es entonces una mera impostura intelectual como el engaño de Sokal. Si el cambio climático lleva a los resultados catastróficos que algunos científicos hoy consideran abiertamente (Kulp y Strauss, 2019; Lenton et al., 2019; Lynas, 2020; 
Moses, 2020; Raymond et al., 2020; Wang et al., 2019; Xu et al., 2020; Yumashev et al., 2019), estos economistas neoclásicos serán cómplices de causar la mayor crisis, no solo en la historia del capitalismo, sino potencialmente en la historia de la vida en la Tierra.

\section{ANEXO}

\section{Cuadro 3}

Tabla SM10-1 y otros estudios de economistas

\begin{tabular}{|c|c|c|c|c|c|c|}
\hline $\begin{array}{l}\text { Autores (con } \\
\text { referencias } \\
\text { si estaban } \\
\text { disponibles) }\end{array}$ & Año & $\begin{array}{c}\text { Calentamiento } \\
\left({ }^{\circ} \mathrm{C}\right)\end{array}$ & $\begin{array}{l}\text { Impacto } \\
\text { (\% PIB) }\end{array}$ & Método & $\begin{array}{c}\text { En IPCC } \\
\text { 2014? }\end{array}$ & Cobertura \\
\hline $\begin{array}{l}\text { Nordhaus } \\
\text { (1991) }\end{array}$ & 1991 & 3 & $-0,25$ & Enumeración & No & $\begin{array}{l}\text { Agricultura, silvi- } \\
\text { cultura, demanda de } \\
\text { electricidad, calenta- } \\
\text { miento en el espacio, } \\
\text { Aumento del nivel } \\
\text { del mar }\end{array}$ \\
\hline Cline & 1992 & 2,5 & $-1,1$ & & No & \\
\hline Cline & 1992 & 10 & $-6,0$ & & No & \\
\hline $\begin{array}{l}\text { Nordhaus } \\
\text { (1994b) }\end{array}$ & 1994 & 3 & $-1,3$ & Enumeración & Sí & $\begin{array}{l}\text { Agricultura, demanda } \\
\text { de energía, aumento } \\
\text { del nivel del mar }\end{array}$ \\
\hline $\begin{array}{l}\text { Nordhaus } \\
\text { (1994b) }\end{array}$ & 1994 & 3 & $-3,6$ & $\begin{array}{l}\text { Solicitud a } \\
\text { expertos }\end{array}$ & Sí & Bienestar total \\
\hline $\begin{array}{l}\text { Fankhauser } \\
\text { (1995) }\end{array}$ & 1995 & 2,5 & $-1,4$ & Enumeración & Sí & $\begin{array}{l}\text { Aumento del nivel } \\
\text { del mar, biodiver- } \\
\text { sidad, Agricultura, } \\
\text { silvicultura, pesca, } \\
\text { demanda de electrici- } \\
\text { dad, recursos hídricos, } \\
\text { comodidad, salud } \\
\text { humana, contamina- } \\
\text { ción del aire, desastres } \\
\text { naturales }\end{array}$ \\
\hline Tol (1995) & 1995 & 2,5 & $-1,9$ & Enumeración & Sí & $\begin{array}{l}\text { Agricultura, biodi- } \\
\text { versidad, aumento del } \\
\text { nivel del mar, salud } \\
\text { humana, demanda } \\
\text { de energía, recursos } \\
\text { hídricos, desastres } \\
\text { naturales, comodidad }\end{array}$ \\
\hline $\begin{array}{l}\text { Nordhaus y } \\
\text { Yang (1996) }\end{array}$ & 1996 & 2,5 & $-1,7$ & Enumeración & Sí & $\begin{array}{l}\text { Agricultura, demanda } \\
\text { de energía, aumento } \\
\text { del nivel del mar }\end{array}$ \\
\hline $\begin{array}{l}\text { Plambeck y } \\
\text { Hope (1996) }\end{array}$ & 1996 & 2,5 & $-2,5$ & Enumeración & Sí & $\begin{array}{l}\text { Aumento del nivel } \\
\text { del mar, biodiver- } \\
\text { sidad, Agricultura, } \\
\text { silvicultura, pesca, } \\
\text { demanda de electrici- } \\
\text { dad, recursos hídricos, } \\
\text { comodidad, salud } \\
\text { humana, contamina- } \\
\text { ción del aire, desastres } \\
\text { naturales }\end{array}$ \\
\hline
\end{tabular}




\begin{tabular}{|c|c|c|c|c|c|c|}
\hline $\begin{array}{l}\text { Autores (con } \\
\text { referencias } \\
\text { si estaban } \\
\text { disponibles) }\end{array}$ & Año & $\begin{array}{c}\text { Calentamiento } \\
\left({ }^{\circ} \mathrm{C}\right)\end{array}$ & $\begin{array}{l}\text { Impacto } \\
(\% \mathrm{PIB})\end{array}$ & Método & $\begin{array}{l}\text { En IPCC } \\
2014 ?\end{array}$ & Cobertura \\
\hline $\begin{array}{l}\text { Mendelsohn, } \\
\text { Morrison et al. } \\
(2000)\end{array}$ & 2000 & 2,2 & 0 & Enumeración & Sí & $\begin{array}{l}\text { Agricultura, silvicul- } \\
\text { tura, aumento del ni- } \\
\text { vel del mar, demanda } \\
\text { de energía, recursos } \\
\text { hídricos }\end{array}$ \\
\hline $\begin{array}{l}\text { Nordhaus y } \\
\text { Boyer }(2000)\end{array}$ & 2000 & 2,5 & $-1,5$ & Enumeración & Sí & $\begin{array}{l}\text { Agricultura, aumento } \\
\text { del nivel del mar, } \\
\text { otros impactos del } \\
\text { mercado, salud } \\
\text { humana, comodidad, } \\
\text { biodiversidad, impac- } \\
\text { tos catastróficos }\end{array}$ \\
\hline $\begin{array}{l}\text { Mendelsohn, } \\
\text { Morrison et al. } \\
(2000)\end{array}$ & 2000 & 2,2 & 0,1 & Estadístico & Sí & $\begin{array}{l}\text { Agricultura, silvi- } \\
\text { cultura, demanda de } \\
\text { energía }\end{array}$ \\
\hline Tol (2002) & 2002 & 1 & 2,3 & Enumeración & Sí & $\begin{array}{l}\text { Agricultura, silvicul- } \\
\text { tura, biodiversidad, } \\
\text { aumento del nivel del } \\
\text { mar, salud humana, } \\
\text { demanda de energía, } \\
\text { recursos hídricos }\end{array}$ \\
\hline $\begin{array}{l}\text { Maddison } \\
(2003)\end{array}$ & 2003 & 2,5 & $-0,1$ & Estadístico & Sí & $\begin{array}{l}\text { Consumo de los } \\
\text { hogares }\end{array}$ \\
\hline $\begin{array}{l}\text { Rehdanz y } \\
\text { Maddison } \\
(2005)\end{array}$ & 2005 & 1 & $-0,4$ & Estadístico & Sí & Felicidad declarada \\
\hline Hope (2006) & 2006 & 2,5 & $-0,9$ & Enumeración & Sí & $\begin{array}{l}\text { Aumento del nivel del } \\
\text { mar, biodiversidad, } \\
\text { Agricultura, silvicul- } \\
\text { tura, pesca, demanda } \\
\text { de energía, recursos } \\
\text { hídricos, comodidad, } \\
\text { salud humana, con- } \\
\text { taminación del aire, } \\
\text { desastres naturales }\end{array}$ \\
\hline $\begin{array}{l}\text { Nordhaus } \\
(2006)\end{array}$ & 2006 & 3 & $-0,9$ & Estadístico & Sí & Producto económico \\
\hline $\begin{array}{l}\text { Nordhaus } \\
(2008)\end{array}$ & 2008 & 3 & $-2,6$ & Enumeración & Sí & $\begin{array}{l}\text { Agricultura, aumento } \\
\text { del nivel del mar, } \\
\text { otros impactos del } \\
\text { mercado, salud } \\
\text { humana, comodidad, } \\
\text { biodiversidad, impac- } \\
\text { tos catastróficos }\end{array}$ \\
\hline $\begin{array}{l}\text { Maddison } \\
\text { y Rehdanz } \\
(2011)\end{array}$ & 2011 & 3,2 & $-12,4$ & Estadístico & Sí & Felicidad declarada \\
\hline $\begin{array}{l}\text { Bosello et al. } \\
(2012)\end{array}$ & 2012 & 1,9 & $-0,5$ & EGC & Sí & $\begin{array}{l}\text { Demanda de energía, } \\
\text { turismo, aumento } \\
\text { del nivel del mar, } \\
\text { desbordamiento de } \\
\text { los ríos, Agricultura, } \\
\text { silvicultura, salud } \\
\text { humana }\end{array}$ \\
\hline
\end{tabular}




\begin{tabular}{|c|c|c|c|c|c|c|}
\hline $\begin{array}{l}\text { Autores (con } \\
\text { referencias } \\
\text { si estaban } \\
\text { disponibles) }\end{array}$ & Año & $\begin{array}{c}\text { Calentamiento } \\
\left({ }^{\circ} \mathrm{C}\right)\end{array}$ & $\begin{array}{l}\text { Impacto } \\
\text { (\% PIB) }\end{array}$ & Método & $\begin{array}{c}\text { En IPCC } \\
2014 ?\end{array}$ & Cobertura \\
\hline $\begin{array}{l}\text { (R Roson and } \\
\text { D. van der } \\
\text { Mensbrugghe, } \\
\text { 2012) }\end{array}$ & 2012 & 2,9 & $-2,1$ & EGC & Sí & $\begin{array}{l}\text { Agricultura, aumento } \\
\text { del nivel del mar, } \\
\text { recursos hídricos, } \\
\text { turismo, demanda de } \\
\text { energía, salud huma- } \\
\text { na, productividad del } \\
\text { trabajo }\end{array}$ \\
\hline $\begin{array}{l}\text { Roson y } \\
\text { Mensbrugghe } \\
\text { (2012) }\end{array}$ & 2012 & 5,4 & $-6,1$ & EGC & Sí & $\begin{array}{l}\text { Agricultura, aumento } \\
\text { del nivel del mar, } \\
\text { recursos hídricos, } \\
\text { turismo, demanda de } \\
\text { energía, salud huma- } \\
\text { na, productividad del } \\
\text { trabajo }\end{array}$ \\
\hline Dellink & 2012 & 2,50 & $-1,1$ & & No & \\
\hline Kemfert & 2012 & 0,25 & $-0,17$ & & No & \\
\hline Hambel & 2012 & 1 & 0,3 & & No & \\
\hline $\begin{array}{l}\text { Burke et al. } \\
\text { (2015) }\end{array}$ & 2015 & 4 & -23 & $\begin{array}{l}\text { Extrapola- } \\
\text { ción lineal }\end{array}$ & No & \\
\hline $\begin{array}{l}\text { Kahn et al. } \\
(2019)\end{array}$ & 2019 & 3,2 & $-7,22$ & $\begin{array}{l}\text { Extrapola- } \\
\text { ción lineal }\end{array}$ & No & \\
\hline
\end{tabular}

Fuente: IPCC (2014, p. SM10-4, Cap. “Sectores Económicos Clave”).

Cuadro 4

Estados Unidos, temperatura promedio, PIB/PEB y datos de población

\begin{tabular}{lllllll}
\hline Estado & $\begin{array}{l}\text { Promedio }{ }^{\circ} \mathrm{C} \\
1971-2000\end{array}$ & PIB 2000 & $\begin{array}{l}\text { Población } \\
2010\end{array}$ & $\begin{array}{l}\text { PIB per } \\
\text { cápita }\end{array}$ & $\begin{array}{l}\text { Desviación } \\
{ }^{\circ} \mathrm{C}\end{array}$ & $\begin{array}{l}\text { PIB per } \\
\text { cápita } \\
\text { Desviación }\end{array}$ \\
\hline Alabama & 17,1 & 119242,4 & 4779736 & $\$ 24,947$ & 5,6 & $-\$ 8.259$ \\
Arizona & 15,7 & 164611,6 & 6392017 & $\$ 25,753$ & 4,2 & $-\$ 7.454$ \\
Arkansas & 15,8 & 68770 & 2915918 & $\$ 23,584$ & 4,3 & $-\$ 9.622$ \\
California & 15,2 & 1366561 & 37253956 & $\$ 36,682$ & 3,7 & $\$ 3.476$ \\
Colorado & 7,3 & 180605,5 & 5029196 & $\$ 35,911$ & $-4,2$ & $\$ 2.705$ \\
Connecticut & 9,4 & 165898,7 & 3574097 & $\$ 46,417$ & $-2,1$ & $\$ 13.210$ \\
Delaware & 12,9 & 43389,4 & 897934 & $\$ 48,321$ & 1,4 & $\$ 15.115$ \\
Florida & 21,5 & 489488,1 & 18801310 & $\$ 26,035$ & 10 & $-\$ 7.172$ \\
Georgia & 17,5 & 307611,6 & 9687653 & $\$ 31,753$ & 6 & $-\$ 1.454$ \\
Idaho & 6,9 & 37992,8 & 1567582 & $\$ 24,237$ & $-4,6$ & $-\$ 8.970$ \\
Illinois & 11 & 487212,7 & 12830632 & $\$ 37,973$ & $-0,5$ & $\$ 4.766$ \\
Indiana & 10,9 & 203052,9 & 6483802 & $\$ 31,317$ & $-0,6$ & $-\$ 1.890$ \\
Iowa & 8,8 & 93028,6 & 3046355 & $\$ 30,538$ & $-2,7$ & $-\$ 2.669$ \\
Kansas & 12,4 & 85853,2 & 2853118 & $\$ 30,091$ & 0,9 & $-\$ 3.115$ \\
Kentucky & 13,1 & 114293,2 & 4339367 & $\$ 26,339$ & 1,6 & $-\$ 6.868$ \\
Louisiana & 19,1 & 132809,9 & 4533372 & $\$ 29,296$ & 7,6 & $-\$ 3.910$ \\
Maine & 5 & 36841 & 1328361 & $\$ 27,734$ & $-6,5$ & $-\$ 5.472$ \\
Maryland & 12,3 & 192106,3 & 5773552 & $\$ 33,274$ & 0,8 & $\$ 67$ \\
Massachusetts & 8,8 & 289926,5 & 6547629 & $\$ 44,280$ & $-2,7$ & $\$ 11.073$ \\
Michigan & 6,9 & 351572,7 & 9883640 & $\$ 35,571$ & $-4,6$ & $\$ 2.365$ \\
Minnesota & 5,1 & 189964,8 & 5303925 & $\$ 35,816$ & $-6,4$ & $\$ 2.609$
\end{tabular}




\begin{tabular}{|c|c|c|c|c|c|c|}
\hline Estado & $\begin{array}{l}\text { Promedio }{ }^{\circ} \mathrm{C} \\
1971-2000\end{array}$ & PIB 2000 & $\begin{array}{l}\text { Población } \\
2010\end{array}$ & $\begin{array}{l}\text { PIB per } \\
\text { cápita }\end{array}$ & $\begin{array}{l}\text { Desviación } \\
{ }^{\circ} \mathrm{C}\end{array}$ & $\begin{array}{l}\text { PIB per } \\
\text { cápita } \\
\text { Desviación }\end{array}$ \\
\hline Mississippi & 17,4 & 65645,9 & 2967297 & $\$ 22,123$ & 5,9 & $-\$ 11.083$ \\
\hline Missouri & 12,5 & 187296,8 & 5988927 & $\$ 31,274$ & 1 & $-\$ 1.933$ \\
\hline Montana & 5,9 & 21885,2 & 989415 & $\$ 22,119$ & $-5,6$ & $-\$ 11.087$ \\
\hline Nebraska & 9,3 & 56503,7 & 1826341 & $\$ 30,938$ & $-2,2$ & $-\$ 2.268$ \\
\hline Nevada & 9,9 & 76627,1 & 2700551 & $\$ 28,375$ & $-1,6$ & $-\$ 4.832$ \\
\hline New Hampshire & 6,6 & 45225,5 & 1316470 & $\$ 34,354$ & $-4,9$ & $\$ 1.147$ \\
\hline New Jersey & 11,5 & 362006,9 & 8791894 & $\$ 41,175$ & 0 & $\$ 7.969$ \\
\hline New Mexico & 11,9 & 55232,9 & 2059179 & $\$ 26,823$ & 0,4 & $-\$ 6.384$ \\
\hline New York & 7,4 & 838660,3 & 19378102 & $\$ 43,279$ & $-4,1$ & $\$ 10.072$ \\
\hline North Carolina & 15 & 275694,2 & 9535483 & $\$ 28,912$ & 3,5 & $-\$ 4.294$ \\
\hline North Dakota & 4,7 & 17976,1 & 672591 & $\$ 26,727$ & $-6,8$ & $-\$ 6.480$ \\
\hline Ohio & 10,4 & 391137,8 & 11536504 & $\$ 33,904$ & $-1,1$ & $\$ 698$ \\
\hline Oklahoma & 15,3 & 90792,7 & 3751351 & $\$ 24,203$ & 3,8 & $-\$ 9.004$ \\
\hline Oregon & 9,1 & 117258,3 & 3831074 & $\$ 30,607$ & $-2,4$ & $-\$ 2.599$ \\
\hline Pennsylvania & 9,3 & 407652,8 & 12702379 & $\$ 32,093$ & $-2,2$ & $-\$ 1.114$ \\
\hline Rhode Island & 10,1 & 34516,4 & 1052567 & $\$ 32,793$ & $-1,4$ & $-\$ 414$ \\
\hline South Carolina & 16,9 & 115246,8 & 4625364 & $\$ 24,916$ & 5,4 & $-\$ 8.290$ \\
\hline South Dakota & 7,3 & 22690,7 & 814180 & $\$ 27,869$ & $-4,2$ & $-\$ 5.337$ \\
\hline Tennessee & 14,2 & 181629,5 & 6346105 & $\$ 28,621$ & 2,7 & $-\$ 4.586$ \\
\hline Texas & 18,2 & 738871 & 25145561 & $\$ 29,384$ & 6,7 & $-\$ 3.823$ \\
\hline Utah & 9,2 & 70291,8 & 2763885 & $\$ 25,432$ & $-2,3$ & $-\$ 7.774$ \\
\hline Vermont & 6,1 & 18311,9 & 625741 & $\$ 29,264$ & $-5,4$ & $-\$ 3.942$ \\
\hline Virginia & 12,8 & 266886,4 & 8001024 & $\$ 33,357$ & 1,3 & $\$ 150$ \\
\hline Washington & 9,1 & 237831,8 & 6724540 & $\$ 35,368$ & $-2,4$ & $\$ 2.161$ \\
\hline West Virginia & 11 & 42606,9 & 1852994 & $\$ 22,994$ & $-0,5$ & $-\$ 10.213$ \\
\hline Wisconsin & 6,2 & 180539 & 5686986 & $\$ 31,746$ & $-5,3$ & $-\$ 1.460$ \\
\hline Wyoming & 5,6 & 17205,4 & 563626 & $\$ 30,526$ & $-5,9$ & $-\$ 2.680$ \\
\hline Estados Unidos & 11,5 & 10252347 & $3,09 \mathrm{E}+08$ & $\$ 33,206$ & 0,0 & $\$ 0$ \\
\hline
\end{tabular}

\section{REFERENCIAS BIBLIOGRÁFICAS}

Amen, M., Bosman, M. M. et al. (2008). Editorial: The urgent need for global action to combat climate change. Globalizations, 5(1), 49-52.

Arent, D. J., Tol, R. S. et al. (2014a). Key economic sectors and services - supplementary material. En C. B. Field, V. R. Barros, et al. (eds.), Climate change 2014: Impacts, adaptation, and vulnerability. Part $A$ : Global and sectoral aspects. Contribution of working Group II to the fifth assessment report of the intergovernmental panel on climate change. Nueva York: Cambridge University Press.

Arent, D. J., Tol, R. S. et al. (2014b). Key economic sectors and services. En C. B. Field, V. R. Barros, et al. (eds.), Climate change 2014: Impacts, adaptation, and vulnerability. Part A: Global and sectoral aspects. Contribution of working Group II to the fifth assessment report of the intergovernmental panel on climate change (pp. 659-708). Nueva York: Cambridge University Press.

Blatt, J. M. (1979). Investment evaluation under uncertainty. Financial Management, 8(2), 66-81. 
Bosello, F., Eboli, F. et al. (2012). Assessing the economic impacts of climate change. An updated CGE point of view. Working paper.

Burke, M., Hsiang, S. M. et al. (2015). Global non-linear effect of temperature on economic production: Supplementary information. Nature, 527(7577), 235-239.

Cai, Y., Lenton, T. M. et al. (2016). Risk of multiple interacting tipping points should encouragerapid $\mathrm{CO}^{2}$ emission reduction. Nature Climate Change, 6(5), 520-525.

Cline, W. (1996). The impact of global warming on agriculture: Comment. American Economic Review, 86(5), 1309-1311.

Darwin, R. (1999). The impact of global warming on agriculture: A Ricardian analysis: Comment. American Economic Review, 89(4), 1049-1052.

DeCanio, S. J. (2003). Economic models of climate change: A critique. Londres: Palgrave Macmillan.

Fankhauser, S. (1995). Valuing climate change: The economics of the greenhouse. Londres: Earthscan.

Field, C. B., Barros, V. R. et al. (2014). IPCC, 2014: Climate change 2014: Impacts, adaptation, and vulnerability. Part A: Global and sectoral aspects. Contribution of working Group II to the fifth assessment report of the intergovernmental panel on climate change. Nueva York: Cambridge University Press.

Forrester, J. W. (1971). World dynamics. Londres: Wright-Allen Press.

Forrester, J. W. (1973). World dynamics. Londres: Wright-Allen Press.

Friedman, M. (1953). The methodology of positive economics. En M. Friedman (ed.), Essays in positive economics (pp. 3-43). Chicago: University of Chicago Press.

Gelman, A. (2014). A whole fleet of gremlins: Looking more carefully at Richard Tol's twice-corrected paper, "The economic effects of climate change", [https://statmodeling.stat.columbia.edu/2014/05/27/ whole-fleet-gremlins-looking-carefully-richard-tols-twicecorrectedpaper-economic-effects-climate-change/].

Gelman, A. (2015). More gremlins: "Instead, he simply pretended the other two estimates did not exist. That isinexcusable". Statistical Modeling, Causal Inference, and Social Science, [https://statmodeling. stat.columbia.edu/2015/07/23/instead-he-simply-pretended-the-othertwo-estimates-did-not-exist-that-is-inexcusable/].

Gelman, A. (2019). The climate economics echo chamber: Gremlins and the people (including a Nobel prize winner) who support them, [https://statmodeling.stat.columbia.edu/2019/11/01/the-environmentaleconomics-echo-chamber-gremlins-and-the-people-including-a-nobelprize-winner-who-support-them/].

Gills, B. y Morgan, J. (2019). Global climate emergency: After COP24, climate science, urgency, and the threat to humanity. Globalizations, 17(6), 885-902.

Gills, B. (2020). Deep restoration: From the great implosion to the great awakening. Globalizations, 17(4), 577-579. 
Hickel, J. (2018). The Nobel prize for climate catastrophe. Foreign Policy, [https://foreignpolicy.com/2018/12/06/the-nobel-prize-for-climatecatastrophe/].

Hope, C. (2006). The marginal impact of $\mathrm{CO}^{2}$ from PAGE2002: An integrated assessment model incorporating the IPCC's five reasons for concern. Integrated Assessment, 6(1), 19-56.

Kahn, M. E., Mohaddes, K. et al. (2019). Long-term macroeconomic effects of climate change: A cross-country analysis. IMF working papers.

Kaufmann, R. K. (1997). Assessing the DICE model: Uncertainty associated with the emission and retention of greenhouse gases. Climatic Change, 35(4), 435-448.

Kaufmann, R. K. (1998). The impact of climate change on US agriculture: A response to Mendelssohn et al. (1994). Ecological Economics, 26(2), 113-119.

Keen, S. (1995). Finance and economic breakdown: Modeling Minsky's 'financial instability hypothesis'. Journal of Post Keynesian Economics, 17(4), 607-635.

Keen, S. (2011). Debunking economics: The naked emperor dethroned? Londres: Zed Books.

Keen, S. (2017). Can we avoid another financial crisis? (The future of capitalism). Cambridge: Polity Press.

Keen, S., Ayres, R. et al. (2019). A note on the role of energy in production. Ecological Economics, 157, 40-46.

Köhler, P., Hauck, J. et al. (2018). Comment on "Scrutinizing the carbon cycle and $\mathrm{CO} 2$ residence time in the atmosphere" by H. Harde. Global and Planetary Change, 164, 67-71.

Kriegler, E., Hall, J. W. et al. (2009). Imprecise probability assessment of tipping points in the climate system. Proceedings of the National Academy of Sciences, 106(13), 5041-5046.

Kulp, S. A. y Strauss, B. H. (2019). New elevation data triple estimates of global vulnerability to sea-level rise and coastal flooding. Nature Communications, 10(1), 4844.

Lenton, T. y Ciscar, J.-C. (2013). Integrating tipping points into climate impact assessments. Climatic Change, 117(3), 585-597.

Lenton, T. M., Held, H. et al. (2008). Supplement to tipping elements in the earth's climate system. Proceedings of the National Academy of Sciences, 105(6), 1786-1793.

Lenton, T. M., Rockström, J. et al. (2019). Climate tipping points - too risky to bet against. Nature, 575(7784), 592-595.

Lynas, M. (2020). Our final warning: Six degrees of climate emergency. Nueva York: HarperCollins Publishers.

Maddison, D. (2003). The amenity value of the climate: The household production function approach. Resource and Energy Economics, 25(2), $155-175$.

Maddison, D. y Rehdanz, K. (2011). The impact of climate on life satisfaction. Ecological Economics, 70(12), 2437-2445.

Meadows, D. H., Randers, J. et al. (1972). The limits to growth. Barcelona: Signet. 
Mendelsohn, R., Morrison, W. et al. (2000). Country-specific market impacts of climate change. Climatic Change, 45(3), 553-569.

Mendelsohn, R., Schlesinger, M. et al. (2000). Comparing impacts across climate models. Integrated Assessment, 1(1), 37-48.

Mirowski, P. (2020). The neoliberal Ersatz Nobel Prize. En D. Plehwe, Q. Slobodian et al. (eds.), Nine lives of neoliberalism (pp. 219-254). Londres y Nueva York: Verso.

Moses, A. (2020). 'Collapse of civilisation is the most likely outcome': top climate scientists. Voice of Action. Melbourne, Australia.

Musgrave, A. (1990). Unreal assumptions. En J. C. Wood y R. N. Woods (eds.), Economic theory: The F-twist untwisted. Milton Friedman: Critical assessments (pp. 333-342). Londres: Routledge.

Nordhaus, W. (1973). World dynamics: Measurement without data. Economic Journal, 83(332), 1156-1183.

Nordhaus, W. (1991). To slow or not to slow: The economics of the greenhouse effect. Economic Journal, 101(407), 920-937.

Nordhaus, W. (1993). Reflections on the economics of climate change. Journal of Economic Perspectives, 7(4), 11-25.

Nordhaus, W. (1994a). Expert opinion on climatic change. American Scientist, 82(1), 45-51.

Nordhaus, W. (1994b). Managing the global commons: The economics of climate change. Cambridge: MIT Press.

Nordhaus, W. (2007). Economics: Critical assumptions in the Stern review on climate change. Science, 317(5835), 201-202.

Nordhaus, W. (2006). Geography and macroeconomics: New data and new findings. Proceedings of the National Academy of Sciences of the United States of America, 103(10), 3510-3517.

Nordhaus, W. (2008). A question of balance. New Haven: Yale University Press.

Nordhaus, W. (2010). Economic aspects of global warming in a postCopenhagen environment. Proceedings of the National Academy of Sciences of the United States of America, 107(26), 1172-11726.

Nordhaus, W. (2013). The climate Casino: Risk, uncertainty, and economics for a warming world. New Haven: Yale University Press.

Nordhaus, W. (2017). Revisiting the social cost of carbon supporting information. Proceedings of the National Academy of Sciences, 114(7), 1518-1523.

Nordhaus, W. (2018a). Nobel lecture. Climate change: The ultimate challenge for economics, [https://www.nobelprize.org/uploads/2018/10/ nordhaus-slides.pdf].

Nordhaus, W. (2018b). Projections and uncertainties about climate change in an era of minimal climate policies. American Economic Journal: Economic Policy, 10(3), 333-360.

Nordhaus, W., Stavins, R. N. et al. (1992). Lethal Model 2: The limits to growth revisited. Brookings Papers on Economic Activity, 1992(2), 1-43.

Nordhaus, W. y Yang, Z. (1996). A regional dynamic general-equilibrium model of alternative climate-change strategies. American Economic Review, 86(4), 741-765. 
Nordhaus, W. y Boyer, J. G. (2000). Warming the world: Economic models of global warming. Cambridge: MIT Press.

Nordhaus, W. y Moffat, A. (2017). A survey of global impacts of climate change: Replication, survey methods, and a statistical analysis. Discussion Paper No. 2096. Cowles Foundation.

Penn, J. L., Deutsch, C. et al. (2018). Temperature-dependent hypoxia explains biogeography and severity of end-Permian marine mass extinction. Science, 362(6419).

Pindyck, R. S. (2017). The use and misuse of models for climate policy. Review of Environmental Economics and Policy, 11(1), 100-114.

Plambeck, E. L. y Hope, C. (1996). PAGE95: An updated valuation of the impacts of global warming. Energy Policy, 24(9), 783-793.

Quiggin, J. y Horowitz, J. K. (1999). The impact of global warming on agriculture: A Ricardian analysis: Comment. American Economic Review, 89(4), 1044-1045.

Raymond, C., Matthews, T. et al. (2020). The emergence of heat and humidity too severe for human tolerance. Science Advances, 6(19). 1-20.

Rehdanz, K. y Maddison, D. (2005). Climate and happiness. Ecological Economics, 52(1), 111-125.

Romer, P. (2016). The trouble with macroeconomics, [https://paulromer. net/trouble-with-macroeconomics-update/WP-Trouble.pdf].

Roson, R. y Mensbrugghe, D. (2012). Climate change and economic growth: Impacts and interactions. International Journal of Sustainable Economy, 4(3), 270-285.

Sokal, A. D. (2008). Beyond the hoax: Science, philosophy and culture. Oxford: Oxford University Press.

Steffen, W., Rockström, J. et al. (2018). Trajectories of the earth system in the anthropocene. Proceedings of the National Academy of Sciences, 115(33), 8252-8259.

Stern, N. (2007). The economics of climate change: The Stern review. Cambridge: Cambridge University Press.

Swain, D., Singh, D. et al. (2020). Attributing extreme events to climate change: A new frontier in a warming world. One Earth, 2(6), 522-527.

Tol, R. S. (1995). The damage costs of climate change toward more comprehensive calculations. Environmental and Resource Economics, 5(4), 353-374.

Tol, R. S. (2002). Estimates of the damage costs of climate change. Part 1: Benchmark estimates. Environmental and Resource Economics, 21(1), 47-73.

Tol, R. S. (2009). The economic effects of climate change. Journal of Economic Perspectives, 23(2), 29-51.

Tol, R. S. (2014). Correction and update: The economic effects of climate change. Journal of Economic Perspectives, 28(2), 221-226.

Tol, R. S. (2018). The economic impacts of climate change. Review of Environmental Economics and Policy, 12(1), 4-25.

Trenberth, K. E. (1981). Seasonal variations in global sea level pressure and the total mass of the atmosphere. Journal of Geophysical Research, 86(C6), 5238-5246. 
Wang, X. X., Jiang, D. et al. (2019). Extreme temperature and precipitation changes associated with four degree of global warming above preindustrial levels. International Journal of Climatology, 39(4), 1822-1838.

Weitzman, M. L. (2011a). Fat-tailed uncertainty in the economics of catastrophic climate change. Review of Environmental Economics and Policy, 5(2), 275-292.

Weitzman, M. L. (2011b). Revisiting Fat-tailed uncertainty in the economics of climate change. REEP Symposium on Fat Tails, 5(2), 275-292.

$\mathrm{Xu}$, C., Kohler, T. A. et al. (2020). Future of the human climate niche. Proceedings of the National Academy of Sciences, 117(21), 11350-11355.

Yumashev, D., Hope, C. et al. (2019). Climate policy implications of nonlinear decline of Arctic land permafrost and other cryosphere elements. Nature Communications, 10(1), 1900. 